\title{
LA DIMENSIONE TRANSNAZIONALE DEI DIRITTI FONDAMENTALI E LO STATO CONSTITUZIONALE APERTO IN EUROPA
}

\author{
PAOLO RIDOLA ${ }^{1}$
}

\begin{abstract}
RESUMO: O artigo pretende analisar o atual processo de formação de um sistema internacional de proteção dos direitos humanos na União Europeia. Para tanto, inicia demonstrando como as diversas etapas do constitucionalismo europeu corroboraram para que os direitos fundamentais viessem a ser compreendidos como produto da "Modernidade europeia". Em seguida, são estudados os principais elementos formadores do "constitucionalismo multinível" que é responsável pela dinâmica funcional do processo de integração europeia. Ao final, o sistema europeu de proteção dos direitos humanos é questionado em sua capacidade de produzir decisões judiciais eficazes frente às jurisdições nacionais dos Estados constitucionais. PALAVRAS-CHAVES: Estado Constitucional; Integração; União Europeia; Direitos Fundamentais.
\end{abstract}

ABSTRACT: This article intends to analyze the current process of formation of an international system of protection of human rights in the European Union. In this sense, the text starts by showing how the various stages of the European constitutionalism have corroborated to affirm the idea that fundamental rights should be understood as a product of the "European Modernity". Following, we focus on the main elements that form the "multilevel constitutionalism" which is responsible for the functional dynamics of the process of European integration. At the end, the European system of protection of human rights is questioned on its ability in making effective judicial decisions against the national courts of constitutional states.

KEYWORDS: Constitutional State; Integration; European Union; Fundamental Rights.

SUMÁRIO: 1. A Europa como "comunidade de direitos fundamentais" e as características do direito constitucional europeu; 2 . Os direitos fundamentais como produto da "modernidade europeia". Liberdade e dignidade humana na primeira "modernidade europeia"; 3. "Modernidade europeia", constitucionalismo e estatalismo: nas raízes das "tradições constitucionais comuns"; 4. Legados históricos, identidade cultural e pluralismo inclusivo na integração constitucional da Europa; 5. Ordenamento europeu dos direitos e valores fundantes do "direito constitucional europeu"; 6. O "constitucionalismo multi-nível", as razões da integração, as fontes da comparação; 7. O "espaço" e o "tempo" dos direitos fundamentais europeus: identidade cultural e integração social; 8. Rumo a um "sistema" europeu de proteção dos direitos fundamentais?

Artigo recebido em 7.02.2011. Artigo aceito para publicação em 31.05.2011 mediante convite.

${ }^{1}$ Università di Roma "La Sapienza". ridola.ml@tiscali.it 
SUMMARY: 1. Europe as a "community of fundamental rights" and the characteristics of European Constitutional Law; 2. Fundamental rights as a product of "European modernity". Freedom and humang dignity in the first "European modernity"; 3. "European modernity", constitutionalism and statism: In the roots of "common constitutional traditions"; 4. Historical legacies, cultural identity and inclusive pluralism in Europe's constitutional integration; 5. European order of the fouding rights and values of "European Constitutional Law"; 6. "Multi-level constitutionalism", the reasons for integration, the sources for comparison; 7. The "space" and "time" of European fundamental rights: cultural identity and social integration; 8. Towards an European "system" of fundamental rights' protection?

\section{L'EUROPA COME “COMUNITÀ DI DIRITTI FONDAMENTALI” E I CARATTERI DEL DIRITTO COSTITUZIONALE EUROPEO}

II titolo richiede una premessa, necessaria per arginare la critica che esso faccia affiorare una visione troppo ottimistica del processo di integrazione costituzionale in Europa. Si può obiettare invero che, nonostante importanti sviluppi anche recenti, culminati con il completamento delle procedure di ratifica del Trattato di Lisbona, tale processo non potrebbe dirsi esaurito, almeno per chi muova dall'assunto che ciò richieda l'approdo ad un compiuto sistema di statualità federale. Si aggiunge, spingendo lo sguardo all'Europa "allargata" dell'area della Convenzione europea dei diritti dell'uomo, che i vincoli nascenti da questa sarebbero pur sempre quelli derivanti da un trattato fra stati sovrani, e che dunque neppure il "sistema Cedu" sarebbe ancora in grado di sostenere un itinerario di "integrazione" fra gli stati contraenti. Queste obiezioni muovono però per un verso da una visione superata della odierna condizione della statualità in Europa, ancorata alle premesse del "sistema Westfalia" ed al poderoso apparato concettuale del diritto pubblico interno formatosi, in questo contesto, a partire dal XIX secolo, nell'epoca del trionfo dello stato nazione. Per altro verso esse peccano nel proporre una accezione troppo statica, e perciò stesso intrinsecamente contraddittoria, del fenomeno della "integrazione". Che, come ha mostrato la elaborazione teorica di Rudolf Smend, alla quale il termine viene comunemente associato, sebbene non senza frequenti incomprensioni e fraintendimenti, fa balzare in primo piano, rispetto alla tradizionale prospettiva dell'equilibrio ("post-Westfalia") fra gli stati, le dinamiche sociali. Sono queste ultime, invero, ad avere scardinato, fin dai primi decenni del XX secolo, le rassicuranti certezze della dogmatica statualistica, ed è merito della Integrationslehre smendiana avere contribuito, muovendo da queste premesse, a liberare la dottrina della costituzione dalla rigida dicotomia della coppia concettuale autorità/ libertà, ed a segnalare, come più coerente con i nuovi equilibri delle democrazie pluralistiche, la necessità di elaborazioni adeguate al continuo divenire del processo di integrazione fra società e stato ed al fluire dinamico del processo di attualizzazione dei valori costituzionali.

Se di ciò è giocoforza prendere atto, trattandosi di sviluppi che corrispondono a trasformazioni epocali degli ordinamenti politici, sembra arduo negare che la transizione dalla Staatslehre alla Verfassungslehre abbia investito anche il 
giudizio della scienza giuridica sui caratteri del processo di integrazione "costituzionale" nell'Unione Europea. In primo luogo, il carattere graduale del suo rafforzamento, così come si è venuto dipanando nell'arco di oltre mezzo secolo, è perfettamente coerente con il paradigma teorico dell' "integrazione", il quale rinvia, come è noto, ad un processo di unificazione sempre in divenire, certamente più fluido rispetto alle rassicuranti gerarchie della costituzionalità formale cresciute nel quadro della dogmatica statualistica, ma assai più consapevole delle opportunità dispiegate dalla continua attualizzazione dei contenuti di valore della costituzione. In secondo luogo, lo stesso leit motiv degli "stati- signori dei trattati", sul quale è stato per gran parte costruito l'assunto della mancanza di un popolo europeo investito di poteri sovrani, richiede di essere sottoposto ad un attento ripensamento critico, che, senza cedere alla tentazione di frettolose fughe in avanti, non dovrebbe trascurare il dato di fatto difficilmente confutabile, e tra l'altro vistosamente confermato dal travagliato iter del Trattato di Lisbona, di una attenzione ed un coinvolgimento crescenti della sfera pubblica europea sulle grandi scelte del processo di integrazione, le quali sono state non soltanto il frutto di accordi tra governi, ma anche l'esito di procedure "discorsive" e di fora di comunicazione pubblica. Così come non va trascurato - per quel che riguarda più direttamente i temi qui affrontati- che le grandi questioni e le lacerazioni anche profonde che investono l'area dei diritti fondamentali nell'area dell'Unione Europea si collocano all'interno di fori allargati transnazionali che coinvolgono il Parlamento europeo, i parlamenti nazionali, le corti europee ed i "giudici" europei, i partiti, le organizzazioni degli interessi, le comunità religiose, la stampa, l'opinione pubblica.

Anche alla luce di ciò, in terzo luogo, appare ingenuo pretendere di sottoporre il processo di integrazione in atto nell'Unione Europea ad un test condotto secondo le consuete categorie elaborate all'interno dell'esperienza del consolidamento dello stato nazione in Europa, quando si prenda consapevolezza che le grandi partiture del poderoso armamentario concettuale della Staatslehre rischiano di ridursi ad un letto di Procuste, qualora questo venga maneggiato secondo criteri astratti e a-storici, con l'obiettivo aprioristico di ricacciarvi dentro a tutti i costi assetti istituzionali, come quelli sviluppatisi attraverso l'evoluzione dei trattati europei, i quali sembrano offrire resistenza alle elaborazioni della tradizionale dogmatica statualistica. II dibattito in corso da anni, il quale ha visto emergere, con riferimento all'Unione Europea, un ventaglio innovativo di opzioni ricostruttive (unione costituzionale/Verfassungsverbund ${ }^{2}$; commonwealth costituito ${ }^{3}$, ordinamento fondamentale di unione/Unionsgrundordnung ${ }^{4}$, federalismo sopranazionale ${ }^{5}$,

${ }^{2}$ Si v. I. Pernice, Der europäische Verfassungsverbund auf dem Wege der Konsolidierung, in Jahrbuch des öffentlichen Rechts" 48, Tübingen 2000, 205 ss.

${ }^{3}$ Cfr. N. Mac Cormick, La sovranità in discussione, Bologna 2003, 269 ss.

${ }^{4}$ Cfr. D. Th. Tsatsos, Die europäische Unionsgrundordnung, in „EuGRZ“ 1995, 287 ss.

${ }^{5}$ Cfr. A. von Bogdandy, Supranationaler Föderalismus als Wirklichkeit und Idee einer neuen Herrschaftsform. Zur Gestalt der Europäischen Union nach Amsterdam, Baden Baden 1999. 
"sinpoliteia" ${ }^{\prime 6}$ etc.) dimostra con evidenza l'insufficienza di schemi concettuali consolidati e la necessità di opzioni adeguate alla comprensione della realtà in trasformazione. Né è casuale che questo dibattito sia stato in larga misura suscitato dalla reazione all'approccio (sostanzialmente interno alla statualità: Staatenverbund) emblematicamente espresso dal Maastricht-Urteil del Bundesverfassungsgericht del 1993. In definitiva, le incertezze manifestatesi in sede teorica, almeno dopo le riforme di Maastricht, circa la qualificazione del processo di costituzionalizzazione dei trattati sono assai indicative, giacchè né gli apparati ricostruttivi elaborati, su base internazionalistica, dal diritto delle organizzazioni internazionali, né quelli giocati sulla distinzione fra confederazioni di stati e stati federali, risultano più adeguati. Una inadeguatezza -va ribadito con decisione- che è storica piuttosto che astrattamente dogmatica, poiché l'assetto delle Comunità europee prima e dell'Unione Europea poi riflette in primo luogo una inedita dimensione della statualità sconosciuta alla Staatslehre tradizionale, in quanto non più incentrata sulla pienezza della sovranità, ma organizzata su "limitazioni della sovranità". Le quali non vanno considerate necessariamente come un vulnus della pienezza dell'autorità degli stati, ma appaiono sempre più come risorse per accrescerne il raggio di azione in contesti vieppiù caratterizzati da molteplici interdipendenze. In secondo luogo, l'esigenza di tentare nuovi itinerari ricostruttivi è stata dettata dalle stesse condizioni "storiche" degli stadi del processo di integrazione europea, i quali, pur avendo di volta in volta sperimentato soluzioni di tipo pattizio di derivazione internazionalistica o di tipo "prefederale", le hanno tutte rielaborate in modo coerente con modalità "cooperative" dell'azione degli stati sostanzialmente inedite.

Ad una conclusione non diversa, circa l'insufficienza delle categorie interne al "sistema Westfalia" per la comprensione degli strumenti di cooperazione fra gli stati in materia di diritti umani, si perviene anche allargando lo sguardo al variegato spettro delle convenzioni regionali, ed in particolare prendendo in considerazione il sistema di protezione delineato dalla Convenzione europea dei diritti dell'uomo. Sebbene la risposta alle sistematiche violazioni dei diritti umani perpetrate in Europa fino alla conclusione della seconda guerra mondiale abbia trovato sviluppo, anche nel caso della Cedu, attraverso documenti dotati della forza vincolante propria dei trattati internazionali, è affermazione largamente condivisa che la Convenzione presenti caratteri peculiari, non integralmente riconducibili agli schemi consolidati del diritto internazionale pattizio. In primo luogo, non sembra convincente restringere il campo di giudizio sulla natura della Convenzione al profilo dell'accordo fra gli stati contraenti, poiché questa visione unilaterale trascura di dare adeguato risalto ad un obiettivo di trascendimento della statualità che fu comune ai documenti internazionali e regionali di protezione dei diritti umani coevi, i quali furono espressione non solo della volontà di definire uno strumentario di

${ }^{6}$ Cfr. D. Th. Tsatsos, The European Sympolity. Towards a New Democratic Discourse (con prefazione di G. Napolitano), Bruxelles 2009. 
protezione vincolante per gli stati, ma altresì dell'intento di "superare" il piano della statualità sovrapponendo ai livelli nazionali, che la storia aveva dimostrato come tragicamente insufficienti, un livello di protezione più alto. Ciò sembra confermato, tra l'altro, dagli svolgimenti della genesi del "sistema Cedu", i quali testimoniano che la Convenzione del 1950 ebbe origine da una iniziativa di mobilitazione dell'opinione pubblica europea, avviatasi all'inizio del 1948, solo in un secondo momento recepita dalle cancellerie degli stati fondatori.

Non devono essere trascurate, inoltre, le implicazioni delle modifiche successive e dei protocolli aggiuntivi della Convenzione. Soprattutto a seguito del Protocollo n. 11, che ha introdotto il ricorso individuale dinanzi alla Corte Europea, la peculiarità del sistema di protezione assicurato dalla Convenzione esce ulteriormente rafforzata anche in confronto con altre convenzioni regionali sui diritti. II Protocollo n. 11 ha infatti dato forte risalto al ruolo della Corte Europea, ponendo al centro del sistema di protezione un'istanza giurisdizionale chiamata a garantire il rispetto degli obblighi derivanti dalla Convenzione. Si tratta di un esito non trascurabile al fine di proiettare sulla scenario dell'Europa allargata della Convenzione le dinamiche di integrazione nel campo dei diritti fondamentali. La circostanza che la Corte sia divenuta l' Herzstück del "sistema Cedu" ha comportato non soltanto un potenziamento degli obblighi degli stati membri, ma ha dato peculiare risalto al rapporto tra la Corte Europea ed i giudici nazionali, contribuendo ad alimentare fra l'una e gli altri circuiti di comunicazione assai attivi, benché non adeguatamente strutturati. Ed il fatto che la questione degli effetti delle decisioni della Corte europea negli ordinamenti nazionali sia assurta ad elemento centrale del dibattito sul "sistema Cedu", attraversando in modo orizzontale le pur rilevanti differenze riguardanti il rango della Convenzione nel diritto interno, non è senza significato. La vasta eco suscitata da ricorrenti contrasti giurisprudenziali sui temi della privacy, della libertà di coscienza, della bioetica, dei diritti politici, della proprietà dimostra che la controversia sull'autonomia della Corte Europea e, correlativamente, dei giudici nazionali nell'interpretazione della Convenzione ha attivato anch'essa, in definitiva, processi di "integrazione" che hanno uno spessore essenzialmente "culturale", perché rinviano ad un confronto sulla lettura, o forse meglio sulla autocomprensione del patrimonio costituzionale europeo dei diritti, che impegna non solo le istanze giurisdizionali, ma larghi strati di una "società aperta" di interpreti e operatori.

Alla luce di ciò non sembra azzardato inquadrare anche il "sistema Cedu" all'interno di dinamiche di integrazione. Ciò deriva anzitutto dalla collocazione peculiare della Convenzione nel panorama dei trattati multilaterali, dal momento che essa, oltre ad avere ad oggetto relazioni tra stati, regola altresì i rapporti fra gli individui, che invocano il rispetto della Convenzione, e gli stati, che ad esso sono obbligati dal diritto internazionale: un tessuto di relazioni occorre aggiungere- che si traduce in un "sistema" di protezione incentrato nel controllo giudiziario sull'osservanza delle garanzie della Convenzione. 
Si tratta, in definitiva, di un complesso strumentario di protezione dei diritti che, sebbene derivante da un trattato, non configura una mera propaggine della dimensione della statualità, in quanto ha assunto una propria connotazione spiccatamente istituzionale (o ordinamentale) ed ha parimenti conquistato una sua peculiare autonomia -come distinto livello sovrastatale di protezionerispetto ai livelli nazionali. E' importante sottolineare, peraltro, che la dottrina più recente, nel rivendicare il carattere peculiare di trattati siffatti, non sconosciuti nel campo della cooperazione multilaterale in tema di diritti umani e definiti come "law making treaties" o "Gesetzesverträge", ha esattamente messo in luce che la loro peculiarità risiede anzitutto nelle virtualità che essi sono capaci di dispiegare sul terreno dell'interpretazione. Questo sforzo ricostruttivo dimostra che la Convenzione sembra essersi oramai da tempo emancipata da una prospettiva di stampo prettamente internazionalistico. Attraverso un complesso gioco di influenze e di recezioni negli ordinamenti costituzionali degli stati contraenti, si è determinato un intreccio molto stretto fra il diritto convenzionale, le costituzioni nazionali e il diritto dell'Unione Europea. Con la conseguenza che oggi si può sostenere che le garanzie dei diritti fondamentali sono elemento essenziale e irrinunciabile della struttura costituzionale europea, pur essendo distribuite tra sistemi giuridici diversi. Uno di questi è rappresentato per l'appunto dalla Convenzione, divenuta vera e propria "Komplementärverfassung", nella quale gli stati membri hanno "custodito" parte del loro diritto costituzionale materiale, cosicché essa è stata di volta in volta qualificata come parte dell' "ordine pubblico europeo" ", come "costituzione europea dei diritti fondamentali", o infine come "costituzione materiale comune europea" ${ }^{\prime \prime}$. Sforzi significativi, questi, perché testimoniano non solo il tentativo di adeguare le costruzioni della scienza giuridica alla comprensione di una realtà in trasformazione, ma inoltre perché collocano i diritti fondamentali europei al centro non di assetti costituzionali isolati ed incomunicabili, ma di un processo di costituzionalizzazione comunicativo, dinamico e svolgentesi su più livelli.

Da questa premessa discende l'articolazione della relazione, nella quale tenterò di tracciare anzitutto un quadro storico dei presupposti e delle aporie della costruzione di un sistema unitario dei diritti fondamentali nella tradizione giuridica europea, ponendomi alla ricerca delle basi sulle quali poggia la elaborazione di "tradizioni costituzionali comuni" nel senso fatto proprio dall'art. 6 TUE. Successivamente cercherò di passare in rassegna i diversi livelli di protezione dei diritti in Europa e di ricostruire problematicamente le interdipendenze fra i diversi livelli. Una vicenda -è importante precisare subito-

\footnotetext{
${ }^{7}$ Cfr. P. Häberle, Europäische Rechtskultur. Versuch einer Annäherung in zwölf Schritten, Baden Baden 1994, 40.

${ }^{8}$ Cfr. J.A. Frowein, Der europäische Menschenrechtsschutz als Beginn eines europäischen Verfassungsrechtsprechung, in „Juristische Schulung“, 1986, 845 ss.;

${ }^{9}$ Cfr. C. Hillgruber, Staat und Religion, in „Deutsches Verwaltungsblatt“ 1999, 1176.
} 
alla quale le corti europee, le corti costituzionali ed i giudici nazionali hanno certo recato un contributo decisivo, e che tuttavia non può dirsi soltanto una vicenda giurisprudenziale, rimasta circoscritta all'interlocuzione fra élites giudiziarie, poiché ad essa ha preso parte una pluralità di attori, abbracciando nel suo fluire dinamico -e peraltro non privo di momenti di partecipazione forti delle opinioni pubbliche - sia i processi di secolarizzazione in atto nella società europea che le risalenti e le nuove fratture identitarie presenti in essa, così come questo quadro viene riflesso nel confronto fra le giurisprudenze, dai fora di discussione dei parlamenti, dalle riforme dei trattati europei e da quelle che hanno investito le legislazioni nazionali in tema di diritti, dalle modifiche dei cataloghi dei diritti a livello statuale ed infrastatuale.

\section{I DIRITTI FONDAMENTALI COME PRODOTTO DELLA "MODERNITÀ" EUROPEA. LIBERTÀ E DIGNITÀ DELL'UOMO NELLA PRIMA “MODERNITÀ” EUROPEA.}

L'approccio alla comprensione delle dinamiche di integrazione negli ordinamenti costituzionali della democrazia pluralistica come processi intrinsecamente culturali e sociali suggerisce anzitutto di riformulare la collocazione dei diritti all'interno di esse. I diritti sono invero radicati nella cultura giuridica e politica europea, ne sono, più precisamente, elemento costitutivo, considerando come essa si è sviluppata storicamente in una secolare (e intrinsecamente pluralistica) tradizione di pensiero, confluita peraltro, a partire dal XIX secolo e nel quadro dell'esperienza dello stato nazionale, nelle elaborazioni dogmatiche della scienza giuridica. Non si intende affermare con questo che, anche in contesti culturali e geografici Iontani, immagini dell'uomo differenti non abbiano prodotto peculiari visioni della posizione dell'individuo nel gruppo sociale o nella comunità politica, ma si vuole soltanto sottolineare che i diritti, in quanto pilastro delle tradizioni costituzionali comuni europee, sono radicati nelle conquiste del pensiero filosofico occidentale e precisamente nell'idea di fondo, comune alla filosofia della conoscenza così come a quella politico-sociale, che sia nel mondo umano che nell'universo fisico non vi sia posto per il dominio del caso. A ben vedere tutto l'itinerario della formazione di un patrimonio costituzionale europeo in tema di diritti è inseparabile dalla premessa che anche nel mondo umano, così come in quello della natura, sia rintracciabile un ordine derivante da un processo di razionalizzazione. Deriva da ciò l'aver costruito i diritti, in tutto il percorso storico del costituzionalismo moderno, come una barriera invalicabile nei confronti di chi detiene il potere o la forza, precisamente nel senso che costui incontri il proprio limite insuperabile in diritti degli individui, che non sono qualcosa di occasionale, imprevedibile o casuale ma corrispondono ad un'istanza superiore di razionalità.

Questo approccio, inoltre, sembra avere ulteriori implicazioni significative. L'avere disancorato il processo di integrazione, in quanto processo evolutivo della dimensione ordinativa della comunità politica, dal suo esclusivo radicamento nei rapporti di forza degli apparati di potere e della statualità, per 
collocarlo anzitutto nel fluire della società, rafforza e rende ineludibile la scelta di assumere un punto di vista storico, e spinge a scavare nel passato e nelle radici profonde della storia della cultura europea, la quale è stata attraversata da contrasti e da conflitti anche drammatici, ha prodotto nel corso dei secoli immagini dell'uomo, concezioni dell'ordine politico e tradizioni giuridiche diverse, ma è stata allo stesso tempo teatro non solo di scontri, ma di contaminazioni, di migrazioni e di comunicazioni di culture anche nel campo del diritto. In particolare, se si vogliono cogliere in profondità i caratteri del patrimonio europeo dei diritti fondamentali, occorre scavare in quel poderoso processo di trasformazione della società che va oramai comunemente sotto il nome di "modernità" europea ${ }^{10}$. In questa vicenda sono confluite, è innegabile, le influenze della tradizione greco-romana e di quella giudaico-cristiana, così come di essa sono state messe in risalto importanti anticipazioni nell'età medievale, oltre che nella lunga esperienza dello ius commune europeo, in alcune manifestazioni del pensiero di questa età, nella concezione del rapporto fra uomo e cosmo, nella elaborazione dei diritti naturali, nella emersione della libertà di coscienza e dell'idea dei limiti del potere politico. Ma è altrettanto indubbio che con l'affermarsi della "modernità" risalenti suggestioni siano state profondamente rielaborate, e ripensate filosoficamente, nel quadro di una visione nuova ed essenzialmente antropocentrica del mondo, precisamente di quella <scoperta del mondo esteriore e dell'uomo>, già evocata da Burckhardt come contrassegno tipico di un'epoca nuova che si apriva ${ }^{11}$, nella quale l'affacciarsi di una nuova immagine secolarizzata dell'uomo sarebbe stata favorita da un lato dalle nuove conoscenze geografiche, che avrebbero incrinato la certezza della universalità del patrimonio teologico e religioso medievale, e dall'altro dall'affermarsi della concezione eliocentrica del cosmo, la quale avrebbe contribuito in modo prepotente a scuotere i pilastri di una mentalità dogmatica.

In questa cornice, è giocoforza riconoscere che il discorso sui diritti nella cultura europea ha preso le mosse dalla riflessione sull'uomo e sulla dignità umana nel pensiero dell'Umanesimo e del Rinascimento. Questo snodo decisivo degli albori della modernità europea si sviluppò all'insegna della rivalutazione del mondo umano in ogni suo aspetto e della <celebrazione dell'uomo completo, che si misura nel mondo e gode nel mondo>, ed in questa completezza della formazione umana si colgono la direzione di senso di una nuova pedagogia e l'elemento unificante della vocazione dell'uomo e della virtus che questi è chiamato a perseguire. ${ }^{12}$ Ed invero chi ripercorra a

\footnotetext{
${ }^{10}$ Per il concetto di "europäische Moderne" v. R. Koselleck, Futuro passato. Per una semantica dei tempi storici (1979), Genova 1986, passim e 250 ss.; J. Habermas, Il discorso filosofico della modernità, Roma- Bari 1987.

${ }_{11}$ Cfr. J. Burckhardt, La civiltà del Rinascimento in Italia, Firenze 1952, 259 ss.

12 Per un quadro d'insieme della concezione dell'uomo nel pensiero umanistico e rinascimentale v. E. Garin, L'umanesimo italiano (1964), Bari- Roma 1994, passim e 25 ss., e 57 per la citazione; Id., Rinascimento e rivoluzioni. Movimenti culturali dal XIV al XVIII secolo (1975), nuova ediz. a cura di M. Ciliberto, Bari- Roma 2007, 3-38.
} 
volo radente il pensiero italiano dell'Umanesimo e del Rinascimento non ha difficoltà a rintracciarvi non solo una immagine dell'uomo sorretta da una concezione elevata della sua dignità, ma un'attenzione puntuale e profonda per tutto l'ampio spettro delle espressioni della personalità e un inventario di motivi ispiratori che faranno molta strada nel discorso europeo dei diritti e nelle più tarde elaborazioni di esso a partire dall'età del costituzionalismo: dal diritto di ogni uomo all'interiorità, a ritrovare in sé stessi tutta la ricchezza della propria vita interiore (Francesco Petrarca), all'affermazione del primato della volontà come strumento della vocazione dell'uomo alla costruzione della "città terrena" e dell'impegno nella società (Coluccio Salutati), alla scoperta della comunanza dei legami fra gli uomini e della virtù della conservazione della città, premessa di una volontà operante per il bene comune e delle basi ideali di una repubblica (Leonardo Bruni), alla valorizzazione dei beni terreni e delle attività economiche, in quanto alla vera virtus non può restare estranea la fecondità delle opere dell'uomo (Poggio Bracciolini), all'affiorare del tema del lavoro come strumento di appropriazione del mondo da parte dell'uomo (Leon Battista Alberti, Giordano Bruno), all'esaltazione del mondo dei piaceri e della vita mondana contro ogni negazione ascetica (Lorenzo Valla), così come a quella della supremazia del sapere, coltivata nella vita contemplativa, giustificata come <la base più profonda dello stesso operare $>$ (Cristoforo Landino), ed infine all'esaltazione della parola, per <il suo valore di comunicazione, di contatto fra uomini> (Lorenzo Valla, Angelo Poliziano), alla fondazione della politica nella virtù civile (Machiavelli, Guicciardini), che avrebbe profondamente ispirato, a partire dal XVIII secolo, l'elaborazione dell'idea della libertà repubblicana.

In questa rapidissima (ed incompleta) rassegna merita di essere particolarmente segnalato, per la profondità delle suggestioni che fornirà al discorso sui diritti nel patrimonio costituzionale europeo, un aspetto. Va ricordato che la stessa concezione della dignità dell'uomo incontrò, nel pensiero dell'Umanesimo e del Rinascimento, un radicale capovolgimento di prospettiva, perché in esso cominciò a delinearsi una nuova antropologia che rivendicò con forza l'eccellenza della dignità umana, la quale era peraltro radicata in un universo secolarizzato e gradualmente svincolata dal fondamento nella imago Dei, che ne aveva rappresentato la giustificazione ed il motivo conduttore nella imponente riflessione del pensiero cristiano e di quello medievale. Nella celebre Oratio de hominis dignitate di Giovanni Pico della Mirandola, l'inserimento del tema della dignità dell'uomo in un universo secolarizzato giunge a compimento arricchendosi di motivi ulteriori. Per un verso, con Pico la riflessione sulla dignità dell'uomo, non più riflesso di una immagine trascendente e racchiusa in un universo fideistico e perciò stesso esclusivo ma espressione della pluralità delle scelte di vita e della ricchezza delle attività umane, apre la strada alla successiva elaborazione dell'idea della tolleranza. ${ }^{13}$ Per altro

${ }^{13} \mathrm{Si}$ v., per la ricostruzione di questi sviluppi, $R$. Forst, Toleranz im Konflikt. Geschichte, Gehalt und Gegenwart eines umstrittenen Begriffs, Frankfurt a.M. 2003, 128 ss. 
verso il pensiero di Pico, pur non recidendo del tutto i legami fra l'eccellenza dell'uomo e l' imago Dei, preannuncia una nuova concezione della dignità, non più (o non soltanto) "dote" che discende per l'intero genere umano dalla creazione, ma come <via da compiere> e conquista dell'uomo capace di foggiare sé stesso nella forma che egli ha preferito, e perciò stesso come una qualità individualizzata nella pluralità delle scelte. L'idea dell'uomo che non "vive" soltanto, ma "conduce" la propria esistenza, dà risalto alla centralità della sua posizione nel mondo ed allo stesso tempo alla inscindibilità della sua dignità dalle "plurali" espressioni della sua libertà.

3. "MODERNITÀ" EUROPEA, COSTITUZIONALISMO E STATALISMO: ALLE RADICI DELLE "TRADIZIONI COSTITUZIONALI COMUNI".

II pensiero umanistico- rinascimentale apriva dunque scenari nuovi e introduceva ad una vera e propria svolta copernicana nella concezione dell'uomo nell'universo, delineando quella proiezione del destino dell'uomo verso il futuro che ha costituito il tratto caratteristico del passaggio della cultura europea alla modernità. Ed invero il percorso verso la modernità avviatosi in questa cruciale fase di transizione avrebbe prodotto quella <profanizzazione della cultura occidentale> evocata da Max Weber, e precisamente <quel processo di disincantamento per via del quale in Europa una cultura profana è scaturita dal disfacimento delle immagini religiose del mondo ${ }^{14}$. Un processo -occorre aggiungere- dipanatosi nei secoli successivi con lo sviluppo di altri concomitanti fattori destinati a rafforzarsi a vicenda, quali l'affermarsi della libertà di coscienza e dell'idea della tolleranza, la separazione fra la sfera religiosa e quella civile, la formazione del capitalismo e la mobilitazione delle risorse produttive, l'incremento della produttività del lavoro, il sorgere di identità nazionali e di poteri statali accentrati, lo sviluppo della vita urbana e la estensione della educazione scolastica, la secolarizzazione dei costumi e degli stili di vita, ed infine la graduale espansione dei diritti di partecipazione politica. Si tratterà quindi di progressi graduali, e la "modernità" europea si profilerà con caratteri ben definiti solo nei secoli XVII e XVIII, teatro dei grandi sommovimenti dell'età del costituzionalismo. Ma già nel Cinquecento sono riconoscibili i tratti essenziali di questo imponente processo di trasformazione, e la scoperta del "nuovo mondo", il Rinascimento e la Riforma segneranno la soglia epocale del passaggio alla "modernità" europea, percepito peraltro non come uno scansione meramente cronologica ma in un significato oppositivo che ne enfatizzava il valore di svolta racchiuso nell'affermarsi dell'immagine di un uomo che, come si legge in un passo illuminante di Montaigne, non <asservisce> ad altri le sue facoltà e si indirizza ad <amministrare la libertà> nella sua anima ${ }^{15}$. La transizione nella "modernità" europea si accompagna pertanto all'acquisizione diffusa di un'autocoscienza della posizione nuova dell'uomo nell'universo, si embrica in un processo di "autocomprensione"

\footnotetext{
${ }^{14}$ Così J. Habermas, II discorso filosofico della modernità, Roma-Bari 1987, 1.

${ }^{15}$ Cfr. M. de Montaigne, Saggi, ediz. ital. a cura di F. Garavini e S. Solmi, Milano 1992, 1339.
} 
(Selbstverständnis) che segna, a ben vedere, in maniera radicale l'intero sviluppo storico dei diritti nel costituzionalismo europeo, essendo per l'appunto riconducibili all'affiorare di un nuovo senso della soggettività non solo la libertà di coscienza, i diritti individuali e le libertà economiche, ma anche i diritti della persona come soggetto radicato nella realtà dei rapporti di vita e nella dimensione della comunità politica e proiettato verso un disegno di liberazione.

In questa cornice, si deve a Reinhard Koselleck il tentativo più approfondito di riformulare il concetto della "modernità". La ricostruzione di Koselleck si muove tra i due poli delle categorie storiche dello <spazio di esperienza/ Erfahrungsraum> e dell' <orizzonte di aspettativa/ Erwartungshorizont>, ritenute idonee a $<$ tematizzare il tempo storico, in quanto intrecciano tra loro il passato e il futuro $>.{ }^{16}$ Ora, secondo la ricostruzione di Koselleck la divaricazione tra esperienza e aspettativa nell'età moderna è progressivamente aumentata, ed essa <può essere concepita come un tempo nuovo> in quanto le aspettative si sono progressivamente allontanate da tutte le esperienze fatte finora. II momento genetico della "modernità" europea si collocherebbe in questo frangente storico, nel quale per un verso lo spazio di esperienza delle generazioni passate risultava spezzato dall'affermarsi di nuove conquiste nei campi più disparati del pensiero e tutte le aspettative erano divenute insicure ed esposte al rischio continuo del loro oltrepassamento; per altro verso, la comparsa di un nuovo orizzonte di aspettativa associato all'idea del progresso dischiuse <il rischio di un futuro aperto $>$, nel quale <la meta della perfezione fu temporalizzata e inserita nel compimento dell'accadere terreno>. In breve, poiché <l'orizzonte di aspettativa ha assunto un coefficiente di cambiamento che progredisce col tempo>, l'intera storia cominciò ad essere concepita come < un processo di perfezionamento continuo e crescente, che, nonostante tutte le ricadute e le deviazioni, deve essere infine pianificato e realizzato dagli uomini stessi>. ${ }^{17}$ Muovendo da queste premesse, Koselleck costruisce su nuove basi l'idea stessa del progresso propria della modernità: un'idea privata oramai di quei connotati compatti ed universali che lo avevano legato in passato a presupposti di natura escatologica, e che si alimenta invece di molte nuove esperienze particolari ed inedite penetrate in tutti i meandri della vita quotidiana e si incentra nella consapevolezza che l'individuo ha un ruolo di $<$ trasformazione attiva di questo mondo $>$. Appunto in questa cornice è accaduto che i confini dello spazio di esperienza e quelli dell'orizzonte di aspettativa abbiano cominciato a divergere, con il conseguente <rovesciamento di tutte le forme di predizione storica un tempo usuali> e la propensione dell'uomo verso un futuro concepito come in principio <diverso dal passato, e migliore>.

La riflessione sulla "modernità" europea sembra peranto imperniata sulla sottolineatura della capacità costruttiva dell'agire umano nella storia e soprattutto la consapevolezza del rapporto fra l'immagine dell'uomo e il flusso del

\footnotetext{
${ }^{16}$ Cfr. R. Koselleck, Futuro passato cit., 300 ss.

${ }^{17}$ Cfr. R. Koselleck, op. ult. cit., 309 ss. 
divenire storico, due profili che offrono chiavi di lettura particolarmente illuminanti dello sviluppo storico dei diritti nel costituzionalismo europeo. Quest'ultimo ha invero utilizzato e rielaborato un patrimonio risalente di pensiero, ma le radici di esso affondano nella formazione di una immagine dell'uomo proiettata verso il futuro e sopratutto aperta senza pregiudizi a nuove esperienze. $\mathrm{E}$ se di ciò si ritiene ineludibile prendere atto per individuare la direzione di senso nella ricerca dei principi (o delle "tradizioni") costituzionali comuni in un'Europa plurale, non è per sbarazzarsi della storia, ma al contrario per inseguirne in modo consapevole le tracce, rifuggendo dal rischio di uno storicismo banalizzato, che interpreta il retaggio della storia come un accumularsi e stratificarsi indifferenziato di esperienze nel corso del tempo, e perciò stesso, neutralizzando tutti i criteri, finisce per smarrire la direzione di senso, che non può che essere il frutto di un'operazione critica, delle scansioni del divenire storico, chiudendo la storia in un museo e riducendo lo storico a colui che <si lascia scorrere fra le dita la successione dei fatti come un rosario $>{ }^{18}$ Sotto questo profilo, è difficile negare che l'interpretazione così radicale della "modernità" europea additata da Hegel attraverso la chiave di lettura dell'autocoscienza della soggettività continui ad indicare una rotta percorribile per la comprensione degli sviluppi dell'integrazione sopranazionale in Europa, a condizione peraltro di intendere questi sviluppi come fenomeni essenzialmente culturali e di assumere dunque una prospettiva kulturgeschichtlich. Questo approccio appare particolarmente interessante ove si porti l'attenzione alla formazione di un patrimonio di diritti come componente fondamentale dell'identità costituzionale europea. L'aver individuato nella soggettività il principio cardine della modernità anche nel suo significato di autofondazione del divenire storico suggerisce almeno due percorsi interpretativi della collocazione (e della fisionomia) di un patrimonio comune di diritti negli itinerari dell'integrazione costituzionale in Europa: non solo, o non soltanto, in quanto il principio di soggettività, così inteso nella sua proiezione dinamica, costituisce una chiave di lettura della evoluzione delle tavole di valori sottese alle esperienze del costituzionalismo europeo; ma soprattutto in quanto esso spinge a considerare i "diritti fondamentali europei" non come un catasto di documenti costituzionali stratificatisi nello spazio e nel tempo né come un monumento della storia o un patrimonio, interamente prigioniero del suo passato, che registra "culture" accumulatesi nei secoli, ma come un inventario aperto al futuro, nel quale lo "spazio di esperienza" non gioca un ruolo costrittivo, ma si converte continuamente in un "orizzonte di aspettativa", in quanto si fonda su una immagine dell'uomo indissolubilmente legata alla capacità di esercitare una vocazione di trasformazione attiva del mondo, e che si confronta pertanto con le nuove sfide poste dal divenire storico. E per quanto riguarda i "diritti fondamentali europei", questo approccio ha due ordini di implicazioni rilevanti alla luce della condizione storica attuale dei processi di integrazione sopranazionale in Europa, i quali si radicano in una

${ }^{18}$ Così ancora W. Benjamin, Tesi di filosofia della storia, Torino 1978, 86. 
società percorsa da diversità profonde e sottoposta alle sfide di imponenti fenomeni migratori: da un lato la proiezione dei diritti europei (ineludibile se ad essi si vuole conservare una funzione materiale di integrazione) verso nuovi "orizzonti di aspettativa", corrispondenti alla molteplicità delle concezioni del mondo, degli stili di vita, dei bisogni, degli interessi, delle domande di liberazione che provengono dalla società; dall'altro, la consapevolezza che tutto ciò postuli di affidare la funzione di integrazione dei diritti europei a congegni inclusivi e flessibili, quali non sono soltanto quelli della codificazione di cataloghi, del law in the books, ma soprattutto quelli di un law in action, di un diritto vivente attraverso una molteplicità di attori, e richieda la necessità di itinerari argomentativi del giurista adeguati a questa realtà.

\section{RETAGGI STORICI, IDENTITÀ CULTURALE E PLURALISMO INCLUSIVO NELL'INTEGRAZIONE COSTITUZIONALE IN EUROPA}

Si collocano qui, a mio avviso, il passaggio fondamentale e l'aporia di fondo di un discorso sui diritti nella cultura europea. Perché in esso sono convissuti e si sono confrontati problematicamente, e con momenti di tensione forti, due filoni. In primo luogo quello della universalità dei diritti, di un patrimonio di diritti indissolubilmente legato ad un' immagine dell'uomo per sua natura destinata a proiettarsi nel tempo e nello spazio oltre i confini della politica e degli stati nazionali. Questa idea dei diritti è parte integrante della modernità europea, embricata nell'affermazione di un'immagine secolarizzata dell'uomo nei più svariati campi della conoscenza, dell'etica, della politica, dell'economia, e lo sviluppo storico dei diritti in Europa ha rappresentato per l'appunto uno degli approdi più alti della modernità. II secondo filone segue per un lungo tratto di strada, almeno nell'Europa continentale, gli itinerari del liberalismo giuridico ottocentesco, si colloca all'interno della vicenda storica del consolidamento degli stati nazionali ed ha avuto come conseguenza l'embricazione dei diritti nella struttura di questi. L'esito di questa vicenda ha comportato l'introiezione dei diritti nell'ambito di un'organizzazione politica caratterizzata da forti elementi di coesione interna, lungo un percorso che ha finito per piegare in funzione unificante (nel senso anzitutto del rafforzamento degli assetti dell'egemonia borghese) piuttosto che in funzione di crescita del pluralismo il grande laboratorio scaturito in Europa dalle guerre di religione, dal giusnaturalismo e dal contrattualismo sei-settecenteschi, dalle rivoluzioni dell'età del costituzionalismo, dalle dottrine del garantismo nel primo liberalismo europeo dell'Ottocento.

Merita di essere ricordato che fu ancora Hegel, nelle Vorlesungen sulla filosofia della storia, a mettere a fuoco questa aporia della modernità europea, che veniva ricondotta al restringimento degli orizzonti dell'illuminismo giuridico entro i confini della edificazione della statualità. Un'aporia consistente nella trasformazione di diritti universali fondati su basi giusrazionalistiche in situazioni soggettive legittimate da un diritto posto da un potere supremo, così come nella conversione di un modello di ordine politico sorretto da un grande progetto di liberazione e da una immagine dell'uomo proiettata verso il futuro 
nella supremazia dello stato e del suo diritto. Riprendendo ancora la dicotomia concettuale di Koselleck, l' "orizzonte di aspettativa" veniva sciolto dai suoi legami con uno "spazio di esperienza" radicato nella tradizione, ma allo stesso tempo veniva cristallizzato attraverso la "im-posizione" del diritto da parte di un potere divenuto supremo.

Alla torsione degli originari motivi ispiratori dell'ideologia del costituzionalismo nello statalismo è corrisposto un faticoso processo di introiezione dei diritti, che non è restato circoscritto al paradigma della edificazione della statualità né ai suoi legami con le esigenze dello stato monoclasse borghese, ma ha continuato ad ispirare la riflessione sul rapporto fra diritti e coesione della comunità politica nelle prime esperienze del costituzionalismo europeo del Novecento. Dal legame con l'esperienza dello stato nazionale sono derivati due ordini di conseguenze: in primo luogo, la formazione di una dogmatica giuridica (rectius: di dogmatiche) dei diritti, le quali non hanno potuto non risentire delle particolarità e dei contesti storico-costituzionali che hanno fatto da sfondo alla loro elaborazione; ed inoltre la progressiva torsione statualistica dei diritti, una prospettiva "introversa" che è stata condizionata dall'egemonia del modello dello stato nazione in Europa ed ha lasciato peraltro una traccia profonda negli indirizzi del pensiero costituzionale della prima metà del Novecento, i quali, anche perché tanto assorbiti dalla riflessione sulla categoria del "politico" nella democrazia di massa, non sono mai riusciti a recidere del tutto il radicamento nei destini dello stato nazionale e sono rimasti, per così dire, a mezzo del guado fra la continuità con la Staatslehre del tardo liberalismo giuridico ottocentesco e la fondazione di una Verfassungslehre embricata nel pluralismo sociale prima che sui rapporti di dominio ricostruiti secondo i canoni propri della scienza politica novecentesca.

Al fine di comprendere perché i diritti siano collocati sempre più al centro del discorso sull'integrazione costituzionale in Europa, ma allo stesso tempo perché essi siano vieppiù divenuti terreno di contrasti e di contrapposizioni accese nella Öffentlichkeit europea, dai temi della bioetica a quelli della laicità, dai diritti politici ai diritti della concorrenza ai diritti sociali, dalla protezione di sfere identitarie all' allargamento dei diritti di cittadinanza nella realtà multiculturale, è necessario pertanto aver presente che lo sviluppo storico dei diritti fondamentali in Europa disegna un itinerario complesso e non privo di contraddizioni, che è proceduto tra universalismo e particolarismo e tra pretese di esclusione e chances di inclusione. Lungo la traiettoria di questa linea di tensione, l'Europa è apparsa come il luogo di confluenza ed il campo di insediamento di culture formatesi fuori dal continente, ma allo stesso tempo come il terreno di elaborazione di fenomeni (costrittivi) di omologazione e di egemonie culturali. E' significativo che le contraddizioni che hanno accompagnato l'identità culturale europea nella sua storia sui temi del rapporto con l'altro e con il diverso siano già presenti proprio nella cultura dell'illuminismo, come testimonia una delle sue espressioni più alte, le Lettres persanes di Montesquieu: il lungo viaggio del protagonista, il persiano Usbek, 
nell'Europa del Settecento è un travagliato itinerario di formazione alla inedita scoperta di un <paese di eretici> e della molteplicità delle concezioni del mondo nell'impervio tentativo di preservare la propria identità nel contatto con un universo relativistico ${ }^{19}$, e tuttavia la conclusione del romanzo, con la punizione del tradimento della favorita rimasta in patria prigioniera dell' harem, lascia intravedere uno scenario nel quale il "rischiaramento" dal pregiudizio è inseparabile dalla omologazione della diversità culturale. E se si ha presente peraltro che nella riflessione sull' idea di Europa sono confluiti nel tempo il cosmopolitismo illuminista e le radici "culturali" del principio di nazionalità nell'età del romanticismo, si comprende perché da questo itinerario il discorso sui diritti in Europa non possa prescindere, e perché proprio da esso, anzi, debba prendere le mosse.

Questo travagliato itinerario indica che la storia dei diritti fondamentali in Europa riflette, per un verso, gli elementi di unità che nella cultura giuridica europea sono stati "mediati" dall'affermazione dell'immagine dell'uomo coessenziale alla "modernità" europea e intrinsecamente universalistica, e per altro verso essa disegna un tracciato frastagliato, dal momento che la <pluralità dei diritti nazionali è parte dell'identità della cultura giuridica europea $>^{20}$. Una pluralità, occorre aggiungere, che, sebbene per un lungo -e tutt'altro che conchiuso- frangente della storia europea abbia trovato espressione e forza nella realtà dello stato nazionale, non appare convincente declinare soltanto lungo l'asse del monopolio del potere al quale la dimensione della statualità ha dato corpo, perché è in primis radicata in diversità di natura culturale, in modi di sentire complessivamente differenti della società, in esperienze storiche differenti. Tutti fattori, questi, che hanno fatto sì che l'immagine dell'uomo radicata nella modernità europea abbia poi trovato declinazioni differenti nelle diverse realtà storiche dell'Europa plurale, alimentando, su un tronco storico in parte comune, concezioni differenti dei diritti, come una concezione individualistica in Francia, statualistico-organicista in Germania, storicistica nell'esperienza inglese. La relazione dialettica fra unità e pluralità nel discorso europeo dei diritti sembra avere pertanto un significato e una pregnanza non integralmente riducibili al trauma della progressiva dissoluzione dello ius commune europeo nella statualità, avendo trovato il suo fondamento nel fatto che la cultura giuridica europea ha offerto declinazioni differenti di un nucleo di pensiero originario, racchiuso appunto nell'immagine dell'uomo elaborata dalla modernità. La tensione fra unità $\mathrm{e}$ pluralità che attraversa la cultura giuridica europea spiega perché, da un lato, lo sviluppo dei diritti fondamentali in Europa non abbia seguito né un percorso lineare né sia giunto all'approdo di una europäische Öffentlichkeit pacificata dai diritti, la cui storia è percorsa da un susseguirsi, tutt'altro che esaurito, di situazioni di conflitto: si pensi al tema dei "diritti dell'altro", che condizionano pesantemente gli equilibri fra libertà e sicurezza in Europa, così

\footnotetext{
${ }^{19}$ Così, ad esempio, J. Starobinski, Introduzione a Montesquieu, Lettere persiane, Milano 2001, 25.

${ }^{20}$ Così P. Häberle, Europaeische Verfassungslehre, IV ediz., Baden Baden 2006, 113.
} 
come a quello dei diritti sociali, che condiziona in modo altrettanto significativo l'elaborazione delle "politiche" dell'Unione. Ed ancora essa spiega perché, nel dibattito sui diritti nell'integrazione costituzionale europea non sia stato difficile ritrovare le grandi dicotomie che il pensiero giuridico ha elaborato in un lungo arco di tempo: quella fra valenza "oppositiva" e valenza "integrativa" dei diritti, fra potenziale "difensivo" e potenziale di "liberazione", fra funzione di "inclusione" e funzione di "esclusione", fra risorse della "neutralizzazione" e prestazioni unificanti racchiuse in "contenuti di valore". Questo spiega, infine, perché la formazione di un sistema europeo dei diritti, proprio perché articolato sulle "diversità", non possa che svilupparsi in modo flessibile, rifuggendo dallo sforzo di elaborare grandi costruzioni concettuali unitarie: si pensi, solo per fare un esempio, alle differenze, irriducibili ad unità, fra la rule of law anglosassone, le libertés publiques di derivazione francese ed i Grundrechte della tradizione tedesca, o ancora ai significati profondamente differenti della riserva di legge nel panorama costituzionale europeo. Derivano da ciò anche le difficoltà di una "dogmatica" europea dei diritti fondamentali, la quale richiederebbe di pervenire a livelli di astrazione e di generalizzazione fondati in contesti omogenei e coesi, cosicché anche per questo aspetto la condizione metodologica di un "diritto costituzionale europeo" non appare diversa dalla (ed anzi si rivela piuttosto paradigmatica della) esperienza delle "costituzioni del pluralismo", la quale ha fatto emergere con forza le difficoltà di una "dogmatica" dei diritti in contesti "pluralistici".

Sono convinto che, se non si hanno chiare le grandi opzioni storico- culturali, unitarie ed insieme plurali, che si sono mosse sullo sfondo dell'esperienza dello stato nazionale, riesce difficile spiegare la formazione di un sistema europeo di protezione dei diritti fondamentali, ed in particolare come, muovendo dal trionfo del giuspositivismo statualistico e dello <stato di diritto introverso $>^{21}$, l' Europa abbia potuto assumere una propria fisionomia come "comunità di diritti fondamentali" (Grundrechtsgemeinschaft) autonoma dalla matrice statualistica e dal condizionamento determinante della volontà degli stati, e come abbiano potuto prendere l'avvio la formazione di un patrimonio costituzionale comune europeo dei diritti e lo sviluppo, soprattutto sebbene non esclusivamente in via pretoria, di "fori" comuni di interpretazione dei diritti: "motori" di integrazione e di europeizzazione, operanti a vari livelli e destinati a funzionare secondo canoni flessibili, idonei a favorire la formazione di un $<$ Gemeinrechtsdenken> in un'Europa solcata da profonde divisioni storiche. ${ }^{22}$ In questo contesto, assume particolare significato la concettualizzazione della categoria del $<$ Gemeinrecht $>$ di recente riformulata da Peter Häberle. L'esperienza europea dell'integrazione sopranazionale avrebbe messo capo alla formazione di un "diritto comune" destinato a svilupparsi sempre più sul piano dei principi: un diritto <strutturato per principi>, che <mette in comune $>$

${ }^{21}$ Uso la nota formula di E. Forsthoff, Stato di diritto in trasformazione (1964), ediz. ital. a cura di C. Amirante, Milano 1973, 287 ss.

${ }^{22}$ Si v. ancora P. Häberle, Europäische Verfassungslehre, Baden Baden 2006, 115 ss. 
solo quel che è davvero fondamentale, è maneggiato e interpretato undogmatisch, ed in tal modo preservato nel suoi caratteri di diritto "aperto" e "flessibile", inseparabili dal carattere pluralistico dell'identità costituzionale europea. ${ }^{23}$ A questo diritto <sussidiario>, costituito da principi generali, da una materiale Allgemeinheit, non si addicono né l'idea di un diritto im-posto dall'alto, presupposta dalle tradizionali Rechtsquellentheorien di impostazione formalistica, né quella di un sistema gerarchicamente ordinato. Ciò perchè la formazione di un nuovo "diritto comune europeo" consiglia un approccio che superi trasversalmente i confini dei diritti nazionali, e richiede di essere consapevoli che le sue "prestazioni di unità" si svolgono, in modo aperto e flessibile, essenzialmente sul piano delle grandi opzioni di principio che stanno sullo sfondo dei diritti nazionali.

\section{ORDINAMENTO EUROPEO DEI DIRITTI E VALORI FONDATIVI DEL "DIRITTO COSTITUZIONALE EUROPEO"}

Questo approccio allo studio dell'ordinamento europeo dei diritti richiede peraltro un approfondimento. La diversità delle culture e dei diritti nazionali che sono confluiti in esso non comporta invero che questo ordinamento si presenti connotato da una assoluta neutralità rispetto a contenuti di valore. Il carattere pluralistico dell'ordinamento europeo dei diritti non lascia spazio a concezioni unilaterali dell'ispirazione di esso, se si conviene con il vecchio monito del justice Holmes che una costituzione pluralistica <non ha lo scopo di incorporare una particolare teoria $>\mathrm{ma}<$ è destinata a gente dalle vedute sostanzialmente diverse $>^{24}$. E tuttavia esso non si presenta appiattito su una dimensione di assoluto relativismo, indifferente alle molteplici componenti culturali che si intersecano nella società europea. Esso è, in breve, neutrale rispetto alla molteplicità delle concezioni del mondo, ma non rispetto ai contenuti di valore che lo alimentano ${ }^{25}$. La elaborazione in via pretoria di principi dell'ordinamento comunitario muovendo dal tronco originario delle libertà economiche dei trattati istitutivi, l'allargamento del catalogo dei diritti fondamentali nell'ordinamento comunitario favorito dalle riforme dei trattati, la strutturazione su grandi campate di valori nella Carta di Nizza, il complesso gioco di reciproche interferenze fra i diritti comunitari, quelli della CEDU e quelli delle costituzioni nazionali e fra le rispettive corti, e l'intera vicenda della formazione dell'ordinamento europeo dei diritti dimostrano che esso è venuto sempre più assumendo una fisionomia qualificata da un frastagliato mosaico di contenuti di valore presupposti tutti come caratterizzanti l'identità costituzionale europea. Ai fini della costruzione di un ordinamento europeo dei diritti, peraltro, sembra questo un itinerario evolutivo inevitabile. II riferimento a valori fondativi di un'identità comune rappresenta, a ben vedere, una via

\footnotetext{
${ }^{23}$ Cfr. P. Häberle, op. ult. cit., 151 ss.

${ }^{24}$ Così con accenti davvero anticipatori, nella dissenting opinion sulla sentenza Lochner del 1905, O.W. Holmes, Opinioni dissenzienti, a cura di C. Geraci, Milano 1975, 50-51.

${ }^{25}$ Riprendo qui la nota distinzione di K. Hesse, Grundzüge des Verfassungsrechts der Bundesrepublik Deutschland, XX ediz., Heidelberg 1999, 4 s.
} 
obbligata per conferire ad esso una propria autonoma forza, che non potrebbe sorreggersi sulla mera imposizione di vincoli e standard ai diritti nazionali senza essere in grado di assicurare, nel contempo, canali di collegamento flessibili con questi: strumenti di collegamento -occorre aggiungere- derivanti non da criteri di disposizione gerarchica dei diversi livelli dell'ordinamento europeo dei diritti, ma da elementi fondativi della "cultura" costituzionale europea, che conferiscono a questa definiti connotati identitari pur mantenendosi aperti alle "concordanze pratiche" necessarie a preservarne il pluralismo ${ }^{26}$. Muovendo da questa premessa, si colgono le potenzialità racchiuse in un approccio all'ordinamento europeo dei diritti che fa leva sugli spazi di gioco fra i valori fondativi dell'identità costituzionale che lo alimentano. La tendenza a costruire tale ordinamento come un mero riflesso delle "limitazioni" della sovranità degli stati denota invece non soltanto una visione assai riduttiva delle sue potenzialità in contesti di interdipendenze transnazionali, ma altresì un marcato e persistente pregiudizio di stampo giuspositivistico, costruito sulla contrapposizione fra limitazioni della sovranità e corrispondenti "controlimiti", che finisce per sacrificarne, o quanto meno per irrigidirne in una gabbia troppo stretta, il carattere aperto e pluralistico.

Occorre riconoscere che il discorso sui "valori", i quali sono tali perché stratificati nel tessuto della società, sposta il baricentro dell'ordinamento europeo dei diritti dal piano della sovranità degli stati, dei cui poteri decisionali essi rimarrebbero, in una linea di continuità con risalenti approdi dogmatici della scienza giuridica, un effetto riflesso o un prodotto di autolimitazione, su quello della "esperienza vivente" di un diritto che risulta da un complesso gioco di interferenze e di migrazioni in un quadro normativo, non soltanto costituzionale, formatosi su più livelli, infrastatuali, nazionali e sovranazionali, sul quale opera una molteplicità di attori, che comprende i legislatori, i governi, i giudici, ma anche l'opinione pubblica nelle sue svariate articolazioni. Un fenomeno di "integrazione" costituzionale, dunque, che corrisponde all'idea di una democrazia autenticamente "repubblicana", la quale si affida al libero confronto delle forze in campo sul modo di intendere i valori fondativi di un Gemeinwohl prima che su formali regole procedurali. Ed invero focalizzare le prestazioni pluralistiche di una <società aperta> solo sul terreno di una interpretazione aperta della costituzione e dell'allargamento del circolo ermeneutico non deve indurre nel fraintendimento che si pervenga in questo modo a sacrificare alle risorse di pluralismo della costituzione la legittimazione democratica del sistema, e che la moltiplicazione degli interpreti della costituzione finisca per rendere così vischioso il processo democratico da comprometterne la imputabilità ad un demos. Si ripropone qui, nei suoi termini essenziali, la controversia fra concezioni assolute e concezioni pluralistiche della democrazia, tra Volksdemokratie e Bürgerdemokratie. A sottovalutarne il significato profondo

\footnotetext{
${ }^{26}$ Sul concetto di praktische Konkordanz come tipico connotato degli ordinamenti costituzionali pluralistici v. ancora K. Hesse, op. cit., 317 ss.
} 
si trascura di prendere atto che una democrazia pluralistica è "costituita" da varietà di voci e di espressioni, da istanze partecipative e contropoteri diffusi e da una comunicazione pubblica aperta alla legittimazione di molteplici attori. E per chi assuma questo angolo visuale, la "società aperta degli interpreti" si presenta essa stessa come fattore di democrazia e non necessariamente di sfaldamento della legittimazione democratica del sistema costituzionale a vantaggio di élites di interpreti in diversa misura ed a vario titolo qualificati, i quali assumerebbero, sebbene non legittimati, l'egemonia del processo democratico. La "porosità" di questo derivante dalla struttura pluralistica della società, in sintesi, non sembra essere di ostacolo alla imputabilità delle decisioni ad un demos, ma la condizione stessa della legittimazione democratica ascritta ad un demos inclusivo.

Nell'ambivalenza del ruolo giocato dai valori, collocati tra la sfera della $<$ formazione del sél Selbstbildung > e quella della <trascendenza dal sél Selbsttranszendenz>, sta anche, a mio avviso, la problematicità del loro rapporto con l'esperienza storica. Nella storia culturale europea, invero, lo "spazio di esperienza" è intessuto di valori fondativi che ne hanno accompagnato lo sviluppo in un lungo arco temporale. Volendone tentare solo una sommaria ricognizione, dovrebbero essere menzionati almeno il valore della libertà, che ha origini risalenti nel sovvertimento dell'esperienza della schiavitù, e dunque in un progetto di liberazione, quello dell'accettazione della differenza, che nasce dai conflitti religiosi e di coscienza dell'età moderna, quello dell'autodeterminazione e del dominio dell'uomo sulla vita quotidiana, derivato dalle grandi sistemazioni della filosofia europea del XVIII secolo, ed infine il valore della razionalità nella sfera pratica, coessenziale ai percorsi della cultura occidentale. E tuttavia, se lo "spazio di esperienza" del costituzionalismo europeo è popolato di valori immersi nella storia, l'"orizzonte di aspettativa" sorretto da queste tavole di valori non smarrisce per ciò stesso il carattere della proiezione verso il futuro, in funzione della ricerca di equilibri sempre nuovi fra i valori fondativi della cultura costituzionale europea, i quali sono non monodimensionali o totalizzanti ma esposti dinamicamente ad un processo di adeguamenti flessibili. Non sembra dunque di poter ravvisare una contraddizione fra l'approccio storico alla ricostruzione dell'identità costituzionale europea, erroneamente frainteso come una scelta di campo metodologica inevitabilmente vocata alla conservazione o all'immobilismo, ed il profilo evolutivo di questa, nel senso dell'apertura verso sviluppi futuri, quando si muova dalla consapevolezza che i retaggi della tradizione sono rielaborati incessantemente dal fluire delle generazioni, che i caratteri fondamentali che informano l'assetto di una comunità politica non sono il deposito (o il retrobottega) di un antiquarium, ma, appunto, direttrici di orientamento di futuri stadi evolutivi, e che l'approccio storico non comporta la chiusura in una visione degli ordinamenti costituzionali "cristallizzata" nelle tradizioni culturali che li hanno alimentati, perché ogni "esperienza" ripensa e rielabora in modo dinamico e critico il proprio passato e la sua storia. Ed allora sottolineare con forza il carattere storico-evolutivo dell'identità 
costituzionale europea non significa limitarsi a conservare un retaggio immobile nel tempo né ad affastellare le voci di un "patrimonio", ma ripensare le grandi scansioni (ed anche le drammatiche rotture) della storia costituzionale europea, affinché esse ci conducano ad individuare le direttrici di orientamento che guidano l'esperienza vissuta, il Gesamterlebnis, di una comunità politica: "valori" storici, per l'appunto, i quali, secondo l'ambivalenza fra la sfera della libertà e quella della costrizione che ad essi è propria, indirizzano ad allineare l'interpretazione del piano delle norme ad un processo di "autocomprensione" che solo consente di riscoprire le ragioni profonde e la direzione di senso di un vissuto collettivo.

II rapporto fra "valori" dell'identità costituzionale europea e storia si profila problematico anche sotto un altro profilo. Un rapido sguardo retrospettivo sulla storia europea dimostra che il susseguirsi di profonde trasformazioni ha rappresentato il Sonderweg alla costruzione di una identità della cultura europea. Mi riferisco alle grandi rotture della storia di questa, come le guerre di religione, la rivoluzione scientifica, la rivoluzione industriale, le rivoluzioni dell'età del costituzionalismo, l'affermarsi dei processi di democratizzazione, la questione sociale, la fuoriuscita dalle esperienze del totalitarismo. Un itinerario -occorre aggiungere- che ha fatto emergere conflitti laceranti più che momenti di ricomposizione unitaria, con ricadute decisive sulle esperienze costituzionali: i conflitti religiosi nell'età della Riforma, sfociati nella definitiva liquidazione dei grandi universalismi politici medievali e nella frattura della nascita della sovranità degli stati; la tensione fra rivoluzione e principio di nazionalità nel XVIII e XIX secolo, che avrebbe posto le basi dell'antagonismo tra sovranità del popolo e sovranità dello stato; quella fra la "cultura" dell'autonomia individuale e la difesa dell'autodeterminazione dell'individuo nei confronti della dimensione collettiva, e per altro verso la formazione di una "sfera pubblica" assunta come scenario di una politica partecipata ed informata e come fuoriuscita da un privato interamente incluso nella manifestazione del sé; ed infine la tensione fra lo sviluppo capitalistico e gli antagonismi di classe, che avrebbe ispirato le grandi fratture della storia costituzionale europea tra mercato e giustizia sociale, tra libertà ed eguaglianza. E peraltro a chi si arresti a considerare la storia europea solo come un inventario di irrisolte fratture riesce difficile immaginare in che modo una pluralità disomogenea di valori emersi da una storia di tensioni e di conflitti (pluralismo e tolleranza, inclinazione al progresso e al miglioramento delle condizioni di vita, libertà e autodeterminazione, eguaglianza e solidarietà) abbia potuto alimentare un'identità comune, con un percorso travagliato che dalle sintesi universalistiche medievali ha condotto alle fratture della modernità per poi incamminarsi verso il faticoso (e problematico) recupero di un comune sfondo identitario. Il punto è che per un verso i due grandi poli ispiratori della cultura europea della modernità, quello greco-romano e quello giudaico-cristiano, si sono formati prima che l'Europa si delineasse con i connotati di una unità continentale, e che per altro verso dalla rottura della unità originaria della 
cultura europea alimentata nel medioevo dal cristianesimo, e da questo riportata nell'alveo del mondo occidentale attraverso la separazione della chiesa ortodossa e la contrapposizione all'islam, sono derivate divisioni e fratture identitarie profonde che sono parte integrante della storia della modernità. II connotato saliente dell'identità culturale europea sembra peraltro risiedere proprio in questa apparente contraddizione della storia e nella sfida poderosa, della quale essa appare tributaria nei confronti della modernità e grazie alla quale risorse di libertà e di pluralismo hanno potuto produrre integrazione senza unità e costruire una identità sulla differenza. E pertanto, se la storia europea indica che nel corso di essa non è mancata la guida rappresentata dal senso di appartenenza ad una identità comune, ciò sembra da ascrivere ai caratteri che la modernità ha impresso su di essa ed al terreno fertile che essa ha precostituito alla formazione di arene di comunicazione pubblica. All'interpretazione di questa linea evolutiva non si addicono pertanto né una concezione "selettiva/normativa" né una concezione "neutrale" della "cultura" europea, ma una diversa concettualizzazione, la quale muove dal presupposto che è la società stessa a determinare la "sua" cultura attraverso processi di comunicazione, perchè l' uomo, nell'ascrivere a determinati valori i propri comportamenti, è impegnato in un'opera di interpretazione del proprio vissuto nella quale non vive come una monade, ma costruisce la sua esperienza in una cornice di interazione sociale. II punto decisivo sta proprio nelle capacità di integrazione dispiegate nel discorso europeo dei diritti dall'autocomprensione fondata su "valori", che, come si è detto, non è statica, ma evolutiva, perché radicata nella società e perciò stesso intrinsecamente adattabile al mutamento della cultura, dei costumi, del tessuto economico-sociale delle generazioni. Proprio questo approccio, che riconduce il collegamento fra diritti e autocomprensione fondata su valori ad una concezione inclusiva dell'identità costituzionale europea e ne individua come costitutiva e inscindibile la coppia integrazione/ differenza, permette di uscire dal vicolo cieco di un Trennungsdenken degli orizzonti culturali dell'Europa, che tende a focalizzarsi sulle "alternative" piuttosto che sulle "possibilità", e dunque sulle scansioni oppositive che li hanno attraversati piuttosto che sugli adattamenti e sugli equilibri, e che finisce inevitabilmente per ridurre l'identità costituzionale europea ad un "patrimonio", che registra nel conto delle sue disparate componenti vittorie e sconfitte, situazioni di dominio e marginalizzazioni, e che perciò stesso appare intrinsecamente chiuso a favorire il dispiegamento delle chances di vita e della molteplicità delle concezioni del mondo. Al contrario, il processo di autocomprensione dei valori riflette società complesse, le quali provengono da ed attualizzano una lunga storia di differenze e di divisioni e peraltro nelle risorse di un'arena comunicativa rinvengono le ragioni profonde della loro convivenza, e nelle quali il confronto e la competizione producono mutamento e ricerca di equilibri sempre rinnovantisi anziché cristallizzazione di egemonie e situazioni di dominio. 


\section{IL "COSTITUZIONALISMO MULTILIVELLO", LE RAGIONI DELL'INTEGRAZIONE, LE RISORSE DELLA COMPARAZIONE}

Alla luce delle considerazioni fin qui svolte, la formazione di un ordinamento europeo dei diritti fondamentali, costituito dalla confluenza degli ordinamenti nazionali in sistemi sopranazionali di protezione non sovrapponibili dal punto di vista geografico né da quello funzionale, ma tra loro comunicanti (l'Unione Europea, la CEDU, l'OSCE), si profila davvero come un laboratorio di ricerca privilegiato per lo studio degli assetti costituzionali delle società pluralistiche contemporanee, nelle quali imponenti fenomeni di migrazione e di comunicazione fra culture hanno messo in crisi la corrispondenza fra la comunità politica e le compatte fisionomie identitarie dello stato nazione. II problema investe anzitutto la questione della sovranità e della sua "crisi". Ed invero è affermazione ricorrente che la sovranità degli stati non rivesta più i connotati di una assoluta supremazia, ma si misuri con gli spazi di gioco di frammenti di sovranità di altre istituzioni che concorrono con essa. Ne sarebbe derivato un <ordinamento pluralistico> nell'ambito del quale più istituzioni non completamente sovrane concorrono nell'esercizio di poteri ritenuti tipico attributo della sovranità. E sebbene il fondamento dell'efficacia degli atti delle istituzioni sopranazionali si collochi nel quadro dei <sistemi giuridici> degli stati, tali istituzioni avrebbero dato vita ad un nuovo <ordine giuridico>, che non costituisce <una dimensione subordinata dei diritti degli stati> né <un semplice sottosistema del diritto internazionale $>{ }^{27} \mathrm{Ne}$ risulterebbe un quadro di <incroci reciproci> di poteri <autonomi>, che contrassegnerebbe la transizione all'era della <post-sovranità>, nella quale il plusvalore derivante dalla imputazione di poteri sovrani ad un demos si sarebbe dissolto nella distribuzione di competenze fra stati e organizzazioni sopranazionali. ${ }^{28}$ Va precisato che questo approccio merita di essere percorso con cautela, in primo luogo perchè esso sembra delineare fino ad ora una linea di tendenza più che una trasformazione compiuta, ed inoltre perchè gli esiti che esso prefigura non sono privi di interrogativi problematici, i quali riguardano non soltanto il complesso rapporto fra "postsovranità" e democrazia, ma anche le ripercussioni sulla intensità e sulla effettività della tutela dei diritti. E peraltro il quadro che ho sinteticamente riassunto si riflette in modo paradigmatico sui sistemi di protezione di questi. La dilatazione di essa su scala sopranazionale sembra per un verso una conseguenza imposta da questo scenario di trasformazioni, in quanto gli orizzonti della sovranità statale non sarebbero più sufficienti, da soli, ad assicurare risposte a domande di libertà provenienti da società la cui complessità trascende i confini tradizionali delle comunità di appartenenza. Per altro verso, la dimensione transnazionale della protezione dei diritti può operare, in modo sussidiario, come un ammortizzatore delle tensioni e dei conflitti più radicati sul terreno identitario. Cosicché le "concordanze pratiche" fra "istituzioni", sorrette in via sussidiaria da clausole di salvaguardia del

\footnotetext{
${ }^{27}$ Così N. Mac Cormick, op. cit., 258.

${ }^{28}$ Così, in una prospettiva parzialmente critica, D. Grimm, Die Souveränität, Berlin 2009, 98.
} 
maggior livello di protezione, sono in definitiva non solo il portato fisiologico di assetti di tutela dei diritti per i quali la transnazionalità opera come moltiplicatore della complessità, ma un argine alla deriva (patologica) della conflittualità sociale nella autocomprensione dei diritti.

Ciò conduce ad un ulteriore ordine di riflessioni, il quale muove dalla constatazione che la internazionalizzazione del diritto costituzionale ha investito non soltanto la questione della sovranità, ma ha trasformato altresì il ruolo $\mathrm{e}$ la forza normativa delle costituzioni. II processo di trasformazione che ha comportato il passaggio da un "sistema" impermeabile di soggetti investiti della pienezza della sovranità ad un quadro di istituzioni "porose", investite di frammenti di poteri sovrani, trova corrispondenza nella transizione dalla supremazia della costituzione verso un assetto di "costituzioni parziali" tra loro raccordate non sul piano di un ordine gerarchico precostituito, ma su quello della armonizzazione dei rispettivi livelli di contenuto. Inoltre, la crescita delle interdipendenze su scala globale, mentre deriva anche dalla tendenza alla generalizzazione di un patrimonio comune di diritti umani, ha sottoposto a tensioni inedite non solo il raccordo fra la cittadinanza e gli spazi dello stato nazione, ma anche il nesso fra il raggio di azione dei diritti e la dimensione della statualità: un nesso -occorre precisare- che ha caratterizzato l'itinerario storico dei diritti fondamentali, sia che esso venga considerato dall'angolo visuale della pienezza di un potere sovrano che ne circoscrive il limite esterno, sia che esso venga riguardato dall'angolo visuale di fini fondamentali assunti a guida del sistema dei diritti ma inglobati in modo esclusivo in un quadro costituzionale comunque riferito all'identità dello stato nazione. Questo quadro sembra essere invero mutato profondamente, per l'effetto congiunto del venir meno della premessa dell'assolutezza della sovranità statale, sulla quale era imperniata la tradizione dello Staatsrecht, e per il marcato condizionamento della supremazia della costituzione, autentico pilastro fondativo della dottrina costituzionale del Novecento, i cui contenuti manifestano una porosità crescente ai "contesti" all'interno dei quali essa "vive", i quali trascendono oramai i confini degli stati. Sotto questo profilo, se è vero che l'ordinamento europeo dei diritti è in larga misura il prodotto di itinerari giurisprudenziali costruito attraverso il "dialogo" fra giudici nazionali e corti europee, nel quale svolge un ruolo sempre più incisivo la comunicazione delle tecniche argomentative e segnatamente delle operazioni di bilanciamento, sarebbe una conclusione parziale arrestarsi a segnalare la formazione di una koinè dell'argomentazione delle corti in Europa, senza tentare di scavare nelle ragioni profonde di essa, perché "comuni" sono anzitutto le esigenze pluralistiche rispetto alle quali i bilanciamenti sono risultati funzionali. Ed invero se essi, in una prima fase della loro storia, furono funzionali al fine di articolare l'interpretazione costituzionale, adeguandola agli scenari di un pluralismo inserito nel contesto identitario tendenzialmente esclusivo della comunità statale, ora hanno trasferito questo ruolo coessenziale a dinamiche pluralistiche sul piano più ampio delle interdipendenze dello stato costituzionale aperto, nel 
quale essi servono ad indirizzare la ulteriore disarticolazione del tessuto della società nel mutato scenario della comunicazione transnazionale.

Se pertanto si vogliono ripercorrere i canali della formazione dell'ordinamento europeo di protezione dei diritti fondamentali, occorre essere consapevoli che in questa materia il ricorso ad approcci di tipo sistematico può risultare fuorviante oltre che problematico, poiché ci troviamo dinanzi ad un assetto sviluppatosi attraverso quel complesso gioco di reciproche interferenze e di interdipendenze che caratterizza la realtà dello stato costituzionale <cooperativo $>0<$ aperto $>.^{29}$ In questa cornice, quello dei diritti fondamentali è stato il terreno privilegiato di processi di scambio, di migrazioni, di recezioni incrociate, che hanno assicurato in modo dinamico l'equilibrio fra la formazione di un diritto costituzionale comune e la preservazione di un profilo identitario plurale. Si comprende allora perché il diritto europeo si presenti come un laboratorio paradigmatico della comparazione giuridica e come banco di prova delle potenzialità del metodo comparativo. Occorre invero prendere atto che il contesto delle interdipendenze fra gli stati e delle contaminazioni tra tradizioni e sistemi giuridici differenti spinge ad un ripensamento serrato del metodo della comparazione giuridica nella direzione della consapevolezza del legame fra i diritti e le culture.

E' merito di Pierre Legrand e soprattutto di Peter Häberle aver avviato un coraggioso rinnovamento del metodo comparativo, il quale muove dalla premessa del carattere intrinsecamente "culturale" della comparazione giuridica. Secondo Häberle l'approccio kulturwissenschaftlich esige un'attenzione peculiare all' "individualità" di una costituzione, la comparazione costituzionale può rivelarsi come uno strumento insostituibile di esso solo a condizione di essere condotta nella consapevolezza della kulturelle Vielfalt che fa da sfondo alle varie esperienze costituzionali. Liberandosi da schematismi e da preoccupazioni di tipo funzionalistico che hanno molto condizionato gli sviluppi della ricerca comparatistica, il pensiero haeberliano sembra restituire alla comparazione un ruolo centrale, identificando in essa l'approdo più alto e lo strato di più intenso spessore della scienza giuridica. Proprio per questa collocazione centrale negli itinerari dell'interpretazione e della scienza giuridica in genere il metodo comparativo non può arrestarsi alla costruzione di schemi, famiglie, formanti, poiché la comparazione si alimenta della tensione dialettica fra l'uno e il diverso, si muove fra i due poli delle Unterschiede e delle Gemeinsamkeiten. E fra questi due poli si sviluppano processi di scambio e interdipendenze, i quali non si esauriscono in un puro esercizio classificatorio, perché attivano un "processo culturale" di Rezeption e di Produktion. ${ }^{30}$

\footnotetext{
${ }^{29}$ Uso le due formulazioni coniate rispettivamente da $P$. Häberle, Die Verfassung des Pluralismus. Studien zur Verfassungstheorie der offenen Gesellschaft, Königstein/Ts. 1980, 287 ss.; e da S. Hobe, Der offene Verfassungsstaat zwischen Souveränität und Interdependenz. Eine Studie zur Wandlung des Staatsbegriffs der deutschsprachigen Staatslehre im Kontext internationaler institutionalisierter Kooperation, Berlin 1998.

${ }^{30}$ Si v. P. Häberle, Verfassungslehre als Kulturwissenschaft, Berlin 200733 ss.
} 
Questo indirizzo metodologico appare di particolare interesse per l'approccio al "diritto costituzionale europeo". Alla luce di esso, infatti, risulta inappagante, da un lato, la tendenza, significativamente coeva allo sviluppo del "sistema Westfalia", che ha identificato l'oggetto della comparazione in un'opera di accatastamento di diritti stranieri, catalogati in modelli o in famiglie, e considerati peraltro come sostanzialmente isolati ed autoreferenziali anche nella dinamica dei loro contatti e dei rapporti reciproci. Per converso, questo indirizzo spinge a rimeditare la tendenza a considerare la comparazione come uno strumento per la formazione di un diritto uniforme. Derivato in origine dal ceppo del giusnaturalismo sei-settecentesco, questo approccio "universalista" del metodo comparativo è stato oggetto di successive riformulazioni che hanno progressivamente preso le distanze dall'ispirazione originaria, ed in tempi recenti è stato ritenuto particolarmente congruente ed attuale nel contesto dei fenomeni di globalizzazione dell'esperienza giuridica, caratterizzati dallo sviluppo di processi di armonizzazione transnazionale del diritto e di vincoli dei diritti nazionali a principi generali elaborati in sedi sovrastatuali. Tuttavia, la percezione del nesso, costitutivo della comparazione, fra diritti e culture ha fornito contributi decisivi alla riflessione sulla inadeguatezza nel metodo comparativo sia di approcci autoreferenziali statocentrati sia di approcci costruiti sulla riconoscibilità di connotati universalistici del diritto. E' stato osservato che, così come ogni cultura è <impregnata di diritto>, la relazione di reciproca dipendenza tra diritto e cultura non deve sorprendere, se si considera che $<i$ giuristi sono essi stessi il prodotto di una determinata società ed operano in una congiuntura spazio-temporale situata culturalmente $>^{31}$ : occorre dunque prendere atto che la comparazione esige <une confrontation radicale avec l'altérité en droit> ${ }^{32}$

Alla luce di queste premesse di carattere metodologico, appare chiaro quali potenzialità possa sviluppare un approccio che punta ad abbandonare $<$ la présomption de similarité pour faire sa place à une véritable experience de la distance et de la différence > nella costruzione del <progetto comparativo> del diritto europeo, all'interno del quale convivono culture radicate in storie diverse e forme di integrazione giuridica sopranazionale. Questo tipo di approccio -è necessario precisare- non deve essere in alcun modo accostato agli indirizzi dottrinali e giurisprudenziali che, preoccupati di salvaguardare un nucleo intangibile della statualità nei processi di cooperazione sopranazionale, fanno leva essenzialmente sulle risorse della sovranità e sul marchio (meramente derivativo) che l'originaria volontà cooperativa manifestata dagli stati, depositari esclusivi di poteri sovrani, lascerebbe impresso in modo indelebile sulle "istituzioni" della sovrastatualità. Sembra invero difficile non riconoscere che queste, benché originate dalla volontà degli stati, abbiano spesso assunto non soltanto una autonoma fisionomia ordinamentale, ma altresì un proprio radicamento "societario" ed una propria legittimazione nell'opinione pubblica europea come luoghi di governo settoriale di una res

\footnotetext{
${ }^{31}$ Cfr. P. Legrand, Le droit comparé, Paris 1999, 5 ss.

${ }^{32}$ Cfr. P. Legrand, Le droit comparé cit., $8 \mathrm{~s}$.
} 
publica pluralista. Altro è invero fondare la ineludibile pluralità dei diritti in Europa sulle "plurali" sovranità degli stati, le quali alla preservazione di essa appresterebbero un corredo inflessibile di controlimiti ad argine di limitazioni comunemente concordate. Altro è muovere dall'assunto che tale pluralità affondi radici in strati profondi della storia delle società europee, che essa abbia un fondamento che è sociale e culturale ad un tempo, al quale la sovranità statale ha offerto certo barriere protettive, da considerare peraltro, nel loro modo di operare come nella loro intensità, non secondo immutabili categorie dogmatiche ma in rapporto al divenire storico. Ragionando di un ordinamento europeo dei diritti fondamentali, questa conclusione può suscitare perplessità, investendo esso un campo presidiato in modo particolarmente intenso dai livelli costituzionali nazionali, i quali, per una tradizione radicata nella storia del costituzionalismo europeo, nel riconoscimento e nella protezione dei diritti hanno identificato una loro vocazione essenziale. Ma se si muove da in orizzonte di osservazione più comprensivo (perché trascendente le dinamiche del rapporto fra enti pienamente sovrani) dei processi di integrazione sopranazionale in Europa, occorre invero prendere atto che la compenetrazione fra i diritti nazionali che essi hanno determinato non ha prodotto un sistema giuridico assolutamente e nella sua "pienezza" uniforme, né è comprensibile peraltro secondo uno schema del rapporto fra livelli statuali e livelli sovrastatuali fondato su moduli di tipo alternativo (aut-aut). Essi hanno invece prodotto l'effetto di mettere in comunicazione fra loro sistemi giuridici differenti in un quadro che è certo sorretto da sfere di competenza prefissate, ma anche ispirato da criteri flessibili e "sussidiari" e inquadrato da comparti sempre più ampi di "diritto per principi" di origine sopranazionale, i quali contemplano margini ampi ad adattamenti ed a "concordanze pratiche". Risiedono in questo, a ben vedere, la collocazione centrale del metodo comparativo nella "costruzione" del diritto europeo e, per quel che interessa il tema dei diritti fondamentali in particolare, le potenzialità (e le sfide) di un "diritto costituzionale comune europeo", poiché in esso diritti e culture giuridiche diverse e comparti di disciplina di differente estensione interagiscono fra loro alimentando una "esperienza". Una esperienza per la comprensione della quale l'approccio della comparazione diviene essenziale, perché essa è per un verso refrattaria al <riduzionismo del diritto alla legge>, ed anzi si dispiega <aldilà delle regole $>^{33}$, e per altro verso non è inquadrabile secondo un criterio di disposizione gerarchica fra ordinamenti senza rischiare di smarrire ad un tempo il significato profondo della sua normatività ed il suo legame con la complessità sociale sottostante.

\section{LO "SPAZIO" E IL "TEMPO" DEI DIRITTI FONDAMENTALI EUROPEI: IDENTITÀ CULTURALE E INTEGRAZIONE SOCIALE}

Le considerazioni fin qui svolte sembrano trovare una conferma particolarmente incisiva nei caratteri di un ordinamento europeo dei diritti, al quale la compenetrazione dei congegni di protezione operanti su più livelli, la

${ }^{33}$ Cfr. P. Legrand, op.ult. cit., $18 \mathrm{~s}$. 
tendenza, diffusa sui diversi livelli, alla formazione di un "diritto per principi" e svolgimenti affidati a processi di interazione fra attori molteplici, istituzionali e non, hanno fatto perdere i connotati di compattezza di un diritto "posto" per configurarlo come un diritto in processu, espresso non da un assetto ordinato di legislatori, ma da fora discorsivi, giurisdizionali e non, di comunicazione pubblica. Se si ripercorre del resto la vicenda della evoluzione di questo ordinamento, è agevole riscontrare come ad essa abbiano contribuito "legislatori" nazionali e sopranazionali, parlamenti e convenzioni ad ampio raggio di legittimazione rappresentativa, istanze giurisdizionali dissenting opinions manifestatesi all'interno di esse, autorità di regolazione, partiti, organizzazioni degli interessi, associazioni e confessioni religiose, settori dell'opinione pubblica europea in uno spazio diffuso di comunicazione pubblica, destrutturato ma non delegittimato, perché popolato di molteplici attori e perciò capace di esprimere freni e contropoteri più forti di quelli che il condizionamento di dinamiche sorrette dal prevalere della regola maggioritaria tende non di rado a sacrificare al livello nazionale.

Di questo ordinamento si tratterà ora di delineare le dinamiche di funzionamento. Occorre considerare anzitutto gli strati temporali attraverso i quali si sono formati i vari "livelli" della articolazione di un sistema europeo dei diritti fondamentali. E' un itinerario nel quale sono confluite e si sono stratificate storicamente esperienze diverse, che dalle dichiarazioni dell'età del costituzionalismo al variegato panorama dei cataloghi dei diritti delle costituzioni nazionali fino alle convenzioni internazionali e regionali del secondo dopoguerra, hanno accompagnato scansioni fondamentali della storia costituzionale comparata, dall'ascesa del giusnaturalismo razionalista e del contrattualismo a quella dello stato nazionale, alla transizione dallo statalismo alla democrazia pluralistica prima e poi dallo "stato di diritto introverso" allo "stato costituzionale aperto". Se si stende lo sguardo sull'ampio spettro temporale delle esperienze costituzionali europee, quello dei diritti fondamentali ci appare non soltanto come il deposito di un patrimonio storico peraltro ricchissimo di suggestioni, ma soprattutto come un terreno sul quale si sono depositati strati temporali diversi, i quali hanno alimentato peraltro processi intrinsecamente evolutivi: sia che essi abbiano preso corpo attraverso operazioni di bilanciamento, le quali recuperano "strati" di contenuti costituzionali che, sebbene cristallizzati in un testo, vengono proiettati nella relazione dialettica tra passato e futuro (la libertà e l'eguaglianza, il profitto e la solidarietà, la libertà e la sicurezza etc.); sia che essi raccolgano in un bloc de constitutionnalité testi risalenti ad epoche ed ispirazioni culturali differenti, che il bloc produce per l'appunto l'effetto di sottrarre al destino altrimenti inevitabile della cristallizzazione in un tempo fisso e determinato per inserirli in dinamiche interpretative non solo più flessibili, ma più comprensive.

La molteplicità degli strati temporali che sono confluiti nell'ordinamento europeo dei diritti si distende peraltro su uno "spazio" esteso, sul quale si dispone anzitutto il livello delle costituzioni nazionali. Esso copre l'area 
dell'Unione Europea e quella, espressiva di un livello di integrazione meno intenso, dell'Europa allargata alla Cedu e all'Osce, e su di esso insistono testi costituzionali risalenti ad epoche molto diverse, frutto di processi costituenti e transizioni differenti, e con cataloghi dei diritti che ne rispecchiano le differenti ispirazioni. In quest'area convivono costituzioni collocate all'interno di esperienze di diritto codificato ed assetti costituzionali sviluppatisi all'interno delle esperienze di common law, caratterizzati da una visione essenzialmente antiformalistica della protezione dei diritti e da congegni particolarmente penetranti di tutela giudiziaria di essi.; cataloghi costituzionali dei diritti di ispirazione complessiva, ampiezza e struttura differenti; costituzioni, come quelle del secondo dopoguerra, le quali poggiano su una Wertordnung finalizzata a realizzare, soprattutto sul terreno dei rapporti economico-sociali, un vigoroso disegno di coesione sociale, e costituzioni di più recente generazione, in particolare quelle espresse dalle transizioni nell'Europa orientale dopo il 1989, le quali hanno declinato il forte risalto dell'intangibilità della dignità dell'uomo con particolare attenzione ai temi della solidarietà nella diversità e della sicurezza nella qualità della vita.

\section{VERSO UN “ SISTEMA" EUROPEO DI PROTEZIONE DEI DIRITTI FONDAMENTALI?}

Del livello sopranazionale di protezione dei diritti fondamentali in Europa, a sua volta articolato in sistemi di diversa intensità, non si offriranno in questa sede un quadro esaustivo né una rassegna compiuta delle elaborazioni giurisprudenziali sui rapporti con le costituzioni e con i diritti nazionali. Si tenterà solo di tratteggiare le "dinamiche" delle relazioni fra i vari livelli. Ciò pone subito dinanzi ad alcuni profili problematici, i quali nascono dalla diversità delle aree sopranazionali di protezione. Per un verso il "sistema" costruito dalla Convenzione Europea del 1950, la quale si prefigge la salvaguardia di uno standard minimo di tutela dei diritti nell'area degli stati contraenti, configurato dalla Corte di Strasburgo, precisamente sul piano della sua <effectiveness>, come <constitutional instrument of European Public Order $>^{34}$. Un "sistema" non statico, ma progressivamente evolutosi in senso giurisdizionale attraverso riforme progressive, come quelle degli anni Novanta in particolare, le quali sono intervenute in modo incisivo sulla razionalizzazione delle procedure, sui sistemi di accesso e sulla struttura del giudizio. Ne è risultato un livello di protezione reso assai incisivo dalla pervasività delle pronunce della Corte, idonee a colpire uno spettro amplissimo di manifestazioni della sovranità degli stati contraenti, da un forte pathos costituzionale di esse ${ }^{35}$, come pure dalle peculiarità drella struttura argomentativa che, in quanto tendenzialmente incentrata su un giudizio secco" di corrispondenza delle misure restrittive degli stati allo standard convenzionale, si è rivelata meno permeabile ad operazioni di bilanciamento.

\footnotetext{
${ }^{34}$ Cfr. Corte Europea dei diritti dell'uomo, Loizidou c. Turchia, 23 marzo 1995, n. 75

${ }^{35}$ Su ciò insiste S. P. Panunzio, I diritti fondamentali e le Corti in Europa, Napoli 2005, spec. 18 ss., 33 ss.
}

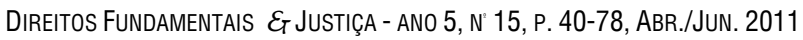


Un profilo, questo, avvertito peraltro come un fattore di difficoltà nel rapporto con la Corte europea da corti costituzionali che attraverso le operazioni di ponderazione hanno riformulato il principio di costituzionalità liberandolo dal rigido schema della corrispondenza fra l'oggetto il parametro del relativo giudizio.

Quanto infine al livello di protezione assicurato dal diritto dell'Unione Europea, anch'esso non va considerato, nel suo sviluppo, come separato dagli altri, con i quali si è misurato in un fitto scambio di interdipendenze e di contaminazioni. In un itinerario al quale ha contribuito in modo rilevante la giurisprudenza "creatrice" della Corte di giustizia, in un confronto serrato non soltanto con le altre corti europee ma altresì con le istituzioni comunitarie e con gli stessi organi costituzionali degli stati. Non va trascurato che l'ampliamento del catalogo dei diritti fondamentali nell'ordinamento comunitario fu preparato a partire dalla fine degli anni Sessanta dalla giurisprudenza della Corte di giustizia, chiamata a rispondere alle sollecitazioni delle corti costituzionali italiana e soprattutto tedesca sulla equivalenza degli standard di tutela dei diritti, e poi progressivamente completato in sedi politiche, come quelle delle riforme dei trattati a partire dall'Atto Unico Europeo del 1986 e delle riforme preparate da convenzioni rappresentative a composizione parlamentare mista (comunitaria e statale). Ne è derivato un progressivo allargamento degli ambiti di tutela dei diritti nell'ordinamento dell'UE, oggi assai più estesi rispetto all'originario impianto produttivistico delle libertà economiche dei trattati istitutivi. Si è così delineato un itinerario sviluppatosi lungo due direttrici di fondo: quella, essenzialmente giurisprudenziale, della "comparazione selettiva (wertende Rechtsvergleichung)" della Cedu e delle tradizioni costituzionali comuni alla luce delle finalità istituzionali dell'Unione, codificata dopo Maastricht dall'art. 6 TUE; e quella della crescente significanza "valoriale" dei diritti europei, testimoniata dalla struttura della Carta di Nizza, la quale ha avuto poi più di un'eco significativa anche nella giurisprudenza comunitaria.

Non va certo trascurato il quadro risultante dal nuovo testo dell'art.6 del TUE dopo le riforme di Lisbona, il quale ha attribuito alla Carta dei diritti fondamentali dell'UE il medesimo valore giuridico dei trattati ed ha previsto l'adesione dell'Unione alla Cedu. E tuttavia non sembra che la nuova formulazione dell'art. 6 , sebbene ricopra un significato politico-costituzionale rilevante nella storia del processo di integrazione, proponendosi di avviare a soluzione nodi assai controversi, sia destinata a sostenere il passaggio da un soft law del sistema di protezione dei diritti fondamentali dell'UE, da maneggiare con i criteri flessibili della "comparazione selettiva", ad un diritto codificato, costituito da un sistema impermeabile di diritti "unionali" razionalizzato da criteri rigidi di relazione con gli altri ordinamenti. Occorre essere consapevoli che, nel caso dell'Unione Europea, ci troviamo dinanzi ad un faticoso processo di costituzionalizzazione che è arduo ricostruire secondo soluzioni di tipo monistico, cosicché lo stesso stadio della "scrittura" di un catalogo di 
diritti resta essenzialmente divaricato, nella sua portata complessiva, da un canone di esclusività degli ordinamenti. Ed in questa ottica il nuovo testo dell'art. 6 inserisce la codificazione di alcune opzioni politico-costituzionali in materia di diritti in un quadro di sostanziale continuità storica e di coerenza con i caratteri peculiari dell'assetto costituzionale dell'UE. Non si intende con ciò sostenere che si tratti di una scelta di apertura alla protezione dei diritti nell'Unione solo di alto significato simbolico, bensì rimarcare la perdurante coessenzialità all'assetto europeo di protezione dei diritti di relazioni interordinamentali sorrette da comparazioni selettive e da veri e propri ammortizzatori delle frizioni fra i diversi livelli. La continuità di questa ispirazione di fondo appare confermata, per quanto riguarda l'UE, proprio dal nuovo testo dell'art. 6 , che imbriglia caute aperture verso elementi meno elastici di codificazione dei diritti in un quadro che resta ancora ricco di fattori di flessibilità. Essi sono riscontrabili in primo luogo nel vincolo dei diritti "unionali" all'estensione delle competenze definite nei trattati, che opera come argine allo sviluppo di un ordinamento generale dei diritti nell'area dell'UE e lascia quindi sostanzialmente immutati i vincoli "selettivi" della dimensione istituzionale/ordinamentale dei diritti "unionali", strumentale cioè al raggiungimento dei fini fondamentali e degli obiettivi dei trattati, sebbene nel quadro del più ampio spettro di essi ricavabile dagli artt. 2 e 3 del TUE. Un complesso gioco di ammortizzatori accompagna, in secondo luogo, la stessa opzione per la adesione alla Cedu, per effetto del vincolo, ribadito anche in questo caso, derivante dalle competenze definite dai trattati e della collocazione della Cedu fra i "principi generali" dell'Unione, che riassorbe pertanto i contenuti della Convenzione in un quadro di tradizioni costituzionali comuni dal quale estrarre un "diritto per principi", rinviando espressamente ad un vincolo alla Cedu di tipo interpretativo, il quale lascia consistenti margini di assestamento fra i due ordinamenti, e soprattutto lascia aperto il problema della priorità delle libertà economiche nel diritto dell'Unione, in quanto esso conceda ad essi <una protezione più estesa> (art. 52.3 della Carta) . Ed infine, quanto ai rapporti fra i diritti dell'Unione ed i livelli nazionali di protezione, il rinvio dell'art. 6 alle disposizioni generali del capo VII della Carta concorre a configurare quelli dell'Unione come diritti "sussidiari", ciò che implica la garanzia della priorità interpretativa del livello di protezione più alto assicurato dagli ordinamenti nazionali (art. 53 della Carta), anch'essa inserita peraltro in una cornice di valutazioni elastiche, secondo il tipico modo di operare del principio di sussidiarietà, riferite alla salvaguardia del $<$ contenuto essenziale> dei diritti riconosciuti e della proporzionalità delle corrispondenti misure restrittive (art. 52 della Carta).

Questi brevi cenni dimostrano che fra i livelli nazionali e quelli sovrastatuali dell'ordinamento europeo dei diritti intercorrono rapporti complessi. Nella dimensione diacronica, anzitutto, dal momento che lo sviluppo di "sistemi" sovrastatuali di protezione si è collocato storicamente a cavallo delle svolte nodali del costituzionalismo europeo del Novecento, fra la stagione delle 
costituzioni del secondo dopoguerra e quella di fine secolo dell'apertura dell'Europa allargata ai principi della democrazia pluralistica. Ciò ha favorito un flusso di relazioni incrociate, in quanto il sistema della Convenzione ha rispecchiato acquisizioni già saldamente radicate nell'esperienza costituzionale europea occidentale e, attraverso le riforme successive, si è progressivamente adattato agli sviluppi di essa, così come il concorrente "sistema" comunitario si è progressivamente modellato su contenuti e congegni di protezione dei diritti già largamente sperimentati. Per altro verso fra le costituzioni europee più recenti è ampiamente riscontrabile la tendenza a costruire l'impianto dei diritti fondamentali modellandolo sulle acquisizioni del "sistema" convenzionale, il recepimento del quale è stato il veicolo principale della penetrazione di formule di riconoscimento e di protezione dei diritti sperimentate dagli ordinamenti costituzionali dell'area europea-occidentale.

Merita di essere sottolineato inoltre che l'ordinamento europeo dei diritti fondamentali, sebbene irriducibile ad un "sistema" unitario e gerarchizzato, negli spazi di gioco tra i diversi livelli assicurati da un complesso ingranaggio di ammortizzatori delle tensioni inevitabili in un assetto non rigidamente strutturato trova peraltro una sua coerenza intrinsecamente pluralistica. Clausole di protezione del contenuto essenziale, tecniche di bilanciamento fra i diritti, canoni di proporzionalità, clausole di salvaguardia del maggior livello di protezione, comparazione selettiva di tradizioni costituzionali comuni, canoni di giudizio cedevoli al "margine di apprezzamento" delle peculiarità nazionali, sono tutte formule ormai entrate a far parte delle dinamiche dell'ordinamento europeo dei diritti attraverso svariate migrazioni da un ordinamento all'altro e da un livello all'altro. Esse delineano peraltro strumenti peculiari per definire l'ambito di operatività dei diritti ai vari livelli nazionali e sovranazionali secondo criteri ed adattamenti flessibili, e per ciò stesso favoriscono contatti, contaminazioni e concordanze tra esperienze diverse. Sarebbe una conclusione unilaterale, peraltro, quella che immaginasse che il complesso gioco ad incastri fra livelli diversi che ho tratteggiato sia comprensibile esclusivamente nella prospettiva di armonizzare la sovranità degli stati con le corrispondenti limitazioni di essa, se non i residui "segmenti" di sovranità di un sistema totalmente destrutturato. Se invero contatti, contaminazioni e concordanze descrivono le peculiari modalità di funzionamento di un ordinamento dei diritti comunicativo che si sviluppa su base comparativa piuttosto che sistematica, occorre domandarsi se ciò non richieda di essere apprezzato anzitutto sulla base di un metro di congruenza con le caratteristiche del tessuto sociale nel quale tale ordinamento si è insediato. Muovendo da questa premessa, non è difficile concludere che contatti, contaminazioni e concordanze corrispondono alle esigenze di società nelle quali le accresciute risorse della comunicazione tra culture si coniugano con la moltiplicazione delle fratture identitarie. Sotto questo profilo, la tutela multilivello dei diritti contribuisce a scardinare ulteriormente lo schema edificato sulla difesa della sfera di libertà privata da attacchi di un soggetto investito di esclusivi poteri sovrani. Essa disaggrega e 
disarticola invero l'opposizione man $v$. state sulla quale essa era stata costruita, in quanto delinea uno scenario nel quale i titolari dei diritti hanno interlocutori differenti, nessuno dei quali si trova peraltro nella condizione di avere l'ultima parola. Questa conclusione può lasciare disorientato chi nutra una fiducia illimitata nelle risorse di garanzia dispiegate dai lineari congegni di autolimitazione del potere sovrano nella tradizionale cornice della legalità statualistica. E tuttavia occorrerebbe domandarsi se le esperienze dello stato legislativo abbiano finito per comportare altresì, nei loro svolgimenti reali e considerate nella prospettiva di lunga durata della storia europea, il sacrificio di spazi di libertà, e se la transizione dal "legalismo" al "costituzionalismo" non abbia peraltro disegnato anche un poderoso processo di allargamento di questi spazi e non abbia già tracciato il percorso nel cui alveo scorrono anche le più recenti esperienze della protezione multilivello dei diritti, una volta che $\mathrm{i}$ confini dello stato costituzionale si siano aperti al circuito delle interdipendenze. Con la formazione di un ordinamento europeo dei diritti non soltanto si è allargato, grazie ad un assetto di ammortizzatori flessibili tra i diversi livelli, il ventaglio delle opportunità nella configurazione e nella protezione di nuovi spazi di libertà, ma soprattutto si è precostituito uno schieramento di "cani da guardia" dei diritti europei, tanto più necessaria in quanto gli attori nazionali sono oramai palesemente inadeguati da soli a far fronte ai rischi aumentati di lesioni e di violazioni dei diritti umani provenienti da attori "privati" transnazionali. Anche sotto questo aspetto, in definitiva, l'ordinamento europeo dei diritti costituisce l'approdo, già largamente preannunciato dalle costituzioni dello stato di democrazia pluralistica, di mutamenti degli assetti ordinamentali corrispondenti a trasformazioni profonde della società, ed il segnale inequivocabile della crisi della dogmatica dei diritti pubblici soggettivi, la cui fortuna nella letteratura italiana ha peraltro di molto travalicato le condizioni storico-costituzionali che ne avevano accompagnato l'elaborazione.

II quadro che ho delineato presenta peraltro zone d'ombra e aspetti contraddittori, i quali lasciano ancora intravvedere come lontana una fase di stabilizzazione. Un primo nodo controverso riguarda i rapporti fra la Cedu e gli ordinamenti nazionali, segnati da divaricazioni significative che squadernano dinanzi a noi soluzioni molteplici. Ciò non costituisce di per sè una patologia in un assetto multilivello, nell'ambito del quale l'anomalia sembra essere rappresentata piuttosto dalla resistenza a "maneggiare" le soluzioni differenti secondo una linea tesa a produrre "concordanze pratiche". Ed invero un assetto multilivello rifugge da schemi omologanti, i quali rischierebbero di imbrigliare le culture giuridiche differenti che hanno condizionato la varietà delle soluzioni riscontrabili : l'incorporazione della Convenzione nel livello costituzionale, come in Austria; la non incorporazione, ma con il contestuale riconoscimento in via pretoria che essa ha rango di diritto costituzionale non scritto, come in Svizzera; la incorporazione nell'ordinamento interno, come è avvenuto nel Regno Unito per effetto dello Human Rights Act del 1998, ma sotto la riserva della parliamentary sovereignty e dunque al di fuori di 
prospettive di tipo gerarchico; la collocazione intermedia fra la costituzione e la legge ordinaria (art. 10 Cost. Spagna, art. 55 Cost. Francia), declinata peraltro con esiti differenti, dal momento che in Spagna la Convenzione è entrata a far parte del bloque de constitucionalidad, mentre in Francia resta ancora ferma la resistenza a far confluire il giudizio di conventionalité in quello di constitutionalité. Ed infine la soluzione dualistica che riconosce alla Convenzione il rango della legge ordinaria che la immette nell'ordinamento nazionale, soluzione condivisa dall'ordinamento tedesco e da quello italiano, sebbene muovendo da approcci sostanzialmente differenti. In Germania la soluzione dualistica ha infatti trovato le sue applicazioni più fertili e significative sul terreno della integrazione materiale e della armonizzazione interpretativa dei contenuti anzichè su quello della disposizione gerarchica delle fonti, ciò che ha implicato il riconoscimento di un vincolo del Bundesverfassungsgericht a <tenere in considerazione> le norme della Convenzione e la relativa giurisprudenza della Corte europea nell'interpretazione del parametro costituzionale interno. Sotto questo profilo, appare particolarmente rilevante il concetto di Völkerrechtsfreundlichkeit, elaborato dalla giurisprudenza del Tribunale costituzionale. Muovendo dalla premessa dell'apertura della Costituzione al diritto internazionale, il Tribunale, pur mantenendosi all'interno di una prospettiva dualistica, e quindi interna al sistema costituzionale del GG, ha assunto un indirizzo argomentativo che privilegia la ricerca di armonizzazioni sul piano dell'interpretazione. Alla stregua di esso, sia la mancanza del confronto con la giurisprudenza di Strasburgo che una $<$ applicazione schematica> che non tenga conto del rango primario assicurato alla Convenzione possono andare contro il sistema costituzionale dei Grundrechte così come contro il principio dello stato di diritto ${ }^{36}$.

Diversamente, la giurisprudenza costituzionale italiana, pur muovendosi come quella tedesca in una cornice di tipo dualistico, evidenzia persistenti rigidità nella gestione del rapporto fra Costituzione e ordinamento convenzionale. Dopo un tormentato itinerario proceduto fra rigida applicazione del criterio gerarchico fondato sulla natura dell'atto interno esecutivo della Convenzione e caute ed isolate aperture all'armonizzazione in via interpretativa, le sentenze n. 348 e n. 349 del 2007 sembrano aver delineato il primo tentativo di dare soluzione organica al problema dell'efficacia della Convenzione e delle pronunce della Corte europea, un tentativo peraltro ancora fortemente condizionato dal ripiegamento delle motivazioni Corte sulla disposizione gerarchica delle fonti, veicolato dal richiamo al nuovo art. $117 \mathrm{co}$. I. Nelle due sentenze si intersecano invero diversi piani dell'argomentazione: la presa d'atto del monopolio della Corte europea nell'interpretazione della Convenzione e allo stesso tempo il richiamo al rango legislativo dell' atto esecutivo interno della Convenzione, che solo il nuovo testo dell'art. $117 \mathrm{co}$. I avrebbe elevato al rango di norma interposta nel giudizio di costituzionalità delle leggi interne

${ }^{36}$ Cfr. BverfGE, 111, 307 (Görgülu- Beschluss, 2004). 
con essa confliggenti, con la conseguente chiusura di ogni possibile varco alla disapplicazione diretta di queste da parte dei giudici nazionali; la sopravvalutazione, con qualche forzatura, della differenza fra lo spessore istituzionale/ordinamentale degli obblighi comunitari e la natura meramente pattizia di quelli nascenti dalla Convenzione; l'assunzione in via esclusiva del parametro dell'art. 117 I co., che mette fuori gioco altri parametri pure fondatamente invocabili, come quelli dell'art. 2, ove inteso come norma di riconoscimento di nuovi diritti fondati non sull'evoluzione della coscienza sociale ma su obblighi internazionali assunti, dell'art. 11, ove si ritenga che la protezione dei diritti umani possa giustificare limitazioni della sovranità fondate sulle finalità da esso richiamate, ed infine dell'art. $10 \mathrm{co}$. 1 , in quanto si ritengano i diritti convenzionali come riproduttivi di consuetudini internazionali ${ }^{37}$. $\mathrm{Ne}$ è derivato un indirizzo non privo di qualche incertezza argomentativa, e peraltro seguito poi da alcune prime applicazioni di questa giurisprudenza ${ }^{38}$, alla stregua del quale la forte rivendicazione delle ragioni del sindacato accentrato contro i rischi di una disapplicazione diffusa da parte dei giudici per vizi di convenzionalità si è coniugato con il rifiuto di argomentare da una prospettiva di "diritto europeo per principi", e di riconoscere alle norme convenzionali anzitutto la natura di "principi generali" di un ordinamento europeo multilivello dei diritti e delle libertà fondamentali. Più di recente, la giurisprudenza del 2007 ha incontrato qualche temperamento, e la Corte sembra riconoscere che il controllo di costituzionalità della legge nazionale per contrasto con il parametro convenzionale interposto viene a configurarsi come l'ultima e del tutto eventuale opzione al termine di un complesso circuito ermeneutico che vede come protagonisti il giudice a quo, chiamato a valutare anzitutto se il contrasto non possa essere risolto per via interpretativa, e la stessa Corte costituzionale, alla quale viene riconosciuta una funzione interpretativa "bifronte", sul versante dell'apprezzamento che il contrasto stesso risulti insanabile alla luce di <un'interpretazione plausibile, anche sistematica> della norma interna rispetto alla norma convenzionale nella lettura datane dalla Corte di Strasburgo, e sul versante della valutazione di un contrasto eventualmente insanabile della norma convenzionale con la Costituzione, che precluderebbe alla prima di assurgere a parametro interposto ${ }^{39}$.

Un secondo nodo controverso riguarda la dottrina dei "controlimiti", la cui elaborazione da parte delle corti costituzionali italiana e tedesca ha in origine contribuito in misura rilevante ad attivare il processo di ravvicinamento dell'ordinamento comunitario ai contenuti ed agli standard di tutela dei diritti fondamentali negli ordinamenti nazionali, ma rischia di concorrere in futuro ad irrigidire le relazioni fra i vari livelli. Nelle giurisprudenze degli ultimi anni,

${ }^{37}$ Soluzione, questa, poi espressamente rifiutata da Corte cost., sent. n. 129 del 2008, ed invece recuperata dalla sent. 311 del 2009, che ha ritenuto pertinente il parametro dell'art. 10 I co. insieme $o$ in alternativa a quello dell'art. 117 I co..

${ }^{38}$ Cfr. Corte cost., sent. n. 39 del 2008.

${ }^{39}$ Cfr. Corte cost., sent. n. 311 del 2009. 
segnali di abbandono della dottrina dei controlimiti in favore di criteri più elastici convivono con indicazioni di segno diverso. Vanno segnalate in particolare quelle che denotano la tendenza a trasferire la dottrina stessa dal piano, assai collaudato nella giurisprudenza delle corti costituzionali, della intangibilità di un nucleo forte degli ordinamenti costituzionali degli stati membri a quello della salvaguardia di un nucleo "costituzionale" di controlimiti propri dei livelli sovrastatuali, nucleo che resterebbe precluso ad operazioni più duttili di ravvicinamento. Mi riferisco, per un verso, alla sentenza Bosphorus della Corte di Strasburgo, che ha affrontato una questione nella quale si fronteggiavano la garanzia della proprietà riconosciuta nel Protocollo n.1 della Convenzione e norme comunitarie attuative di convenzioni internazionali sul trasporto aereo: un contrasto che la Corte europea dei diritti, pur meno attrezzata, per la struttura peculiare del suo giudizio, a ricercare "concordanze pratiche", ha risolto individuando una soluzione di bilanciato equilibrio fra "diritti fondamentali convenzionali e "diritti fondamentali comunitari", e riconoscendo che il rispetto di obblighi internazionali e comunitari degli stati possa essere assunto a fondamento di misure restrittive di diritti convenzionali, a condizione che risulti salvaguardato dal diritto comunitario uno standard comparabile di tutela dei diritti fondamentali, comunque rimesso all'apprezzamento della Corte europea $^{40}$. Un approccio differente sembra ispirare invece la sentenza Kadi della Corte di giustizia del 2008, con la quale è stato annullato un regolamento comunitario in esecuzione di una risoluzione del Consiglio di sicurezza dell'Onu che aveva deciso misure restrittive in campo patrimoniale di contrasto nei confronti del terrorismo internazionale. Nel negare la immunità giurisdizionale del regolamento per la sua riconducibilità allo ius cogens per gli stati derivante da obblighi internazionali, la Corte ha affermato che gli obblighi imposti da un accordo internazionale non possono avere l'effetto di compromettere i principi fondamentali dei trattati, <fra i quali vi è il principio secondo cui gli atti comunitari devono rispettare i diritti fondamentali $>^{41}$. Nonostante l'importante richiamo ai diritti come principi fondamentali del diritto dell'Unione, che ha emblematicamente riguardato peraltro in questo caso libertà attinenti alla sfera patrimoniale, e dunque storicamente lo zoccolo duro del diritto comunitario, la sentenza Kadi solleva qualche interrogativo, laddove essa sembra individuare un nucleo forte di diritti comunitari, i quali operano come "controlimiti" della "costituzione dell'UE" nei confronti degli obblighi internazionali degli stati membri. In questo modo essa lascia intendere peraltro in quale misura l'itinerario argomentativo fondato sui "controlimiti" possa determinare situazioni di stallo nei rapporti fra i diversi livelli e ostacolare la comunicazione fra i medesimi. E del resto l'intera vicenda del rapporto fra "diritti fondamentali

${ }^{40}$ Cfr. Corte europea dei diritti dell'uomo, sentenza 45036/98, Bosphorus Airways V. Irlanda, 30 giugno 2005.

${ }^{41}$ La sentenza Kadi, 3 settembre 2008, cause C-402/05 P e C-415/05 P, può leggersi ora in "Giur. Cost." 2009, 1253 ss., ed ivi un ampio e pregevole commento di $A$. Schillaci, Tutela dei diritti cit., 1255 ss. 
comunitari" e "diritti fondamentali nazionali" dimostra che l'elaborazione della dottrina dei controlimiti abbia costituito un fattore di rigidità nella risoluzione di un rapporto ben altrimenti complesso, i cui passaggi essenziali non sembrano peraltro integralmente riconducibili ad essa: né la partita che si è giocata intorno alla primauté del diritto comunitario direttamente applicabile, la quale si sviluppata in parallelo con la elaborazione "comparativa" di un sistema di diritti fondamentali comunitari, né lo sviluppo delle giurisprudenze nazionali che hanno affermato il principio del livello non equivalente, l'una e l'altro non comprensibili invero al di fuori di un percorso di comunicazione. Ne offrono un esempio, fra l'altro, non isolate pronunce delle corti costituzionali che hanno scelto di percorrere la via della interpretazione del parametro costituzionale interno alla luce dei principi dell'ordinamento comunitario piuttosto che quella additata dalla dottrina dei controlimiti. ${ }^{42}$ Alla quale sembra invece speculare il rifiuto della Corte costituzionale italiana di qualificarsi come giurisdizione nazionale ai fini dell'ammissibilità del sollevamento della pregiudiziale comunitaria. Un rifiuto che non sembra scalfito né dalla ordinanza n. 103 del 2008 , che ha riconosciuto tale ammissibilità con riferimento ai giudizi in via principale e sulla base della <particolare natura di tali giudizi>, che segna un discrimine netto rispetto ai circuiti dell'interpretazione delle norme comunitarie che si sviluppano nell'ambito delle giurisdizioni nazionali, né da qualche ulteriore successiva apertura. Mi riferisco ad una decisione molto recente, con la quale, da un lato la Corte sembra spezzare una lancia in favore della integrazione materiale fra principi costituzionali e principi del diritto dell'UE, laddove si legge che il principio della retroattività della legge penale più mite trova non solo una conferma, ma una <copertura> nell'art. 49 della Carta di Nizza, la cui portata non si esaurisce pertanto nell'offrire un argomento ad adjuvandum dell'interpretazione della Costituzione; dall'altro introduce una significativa smagliatura nella giurisprudenza sulla pregiudiziale comunitaria, laddove si legge che il rinvio pregiudiziale richiesto in via preliminare alla Corte medesima risultava precluso solo in quanto <non necessario, quando il significato della norma comunitaria sia evidente> alla luce di indirizzi consolidati della giurisprudenza comunitaria. ${ }^{43}$ Proprio questa recente contestuale apertura sul fronte del rinvio pregiudiziale e su quello dell'integrazione materiale dimostra per un verso che dietro la controversia sul rinvio si nasconde una questione decisiva, quella della partecipazione delle corti costituzionali ad un circuito comunicativo che già vede pienamente coinvolti i giudizi nazionali e che peraltro, come si è tentato di dimostrare, è parte essenziale della formazione di un diritto costituzionale europeo; e per altro verso spinge nella direzione di un ripensamento critico della dottrina dei controlimiti, dal momento che non vi può essere comunicazione laddove ci si trovi di fronte ad un incastro di rigide

\footnotetext{
${ }^{42}$ Si v., ad es., Corte cost., le sentenze n. 420 del 1994 (in tema di pluralismo dell'informazione) e n. 443 del 1997 (in tema di discriminazioni a rovescio).

${ }^{43}$ Cfr. Corte cost., sent. n. 28 del 2010.
} 
paratie di controlimiti che presidiano in modo impenetrabile ognuno dei piani di un ordinamento europeo dei diritti.

Un terzo nodo problematico riguarda il catalogo dei diritti fondamentali dell'UE. Di esso mi limito a segnalare due aspetti, per il ruolo decisivo che essi giocheranno nel futuro dell'integrazione costituzionale in Europa. L'ordinamento europeo dei diritti fondamentali appare oscillante fra la neutralizzazione delle istanze di libertà provenienti da una società complessa e la costruzione di un assetto ordinato intorno a tavole di valori. Nella prima stagione della giurisprudenza comunitaria le aperture sul fronte dei diritti furono essenzialmente costruite sull'uso giurisprudenziale dei canoni di un mercato aperto e in concorrenza e dei divieti di discriminazione, attraverso una "generalizzazione estensiva" del nucleo delle libertà economiche più che attraverso una "comparazione selettiva" di principi costituzionali comuni. E orientamenti interlocutori della Corte di giustizia, come quello sul caso Grogan, che riguardava il rapporto fra il diritto di associazioni studentesche irlandesi di fare pubblicità agli aborti praticati nel Regno Unito e la libertà professionale del medico, dimostrarono che posizioni di libertà costruite sull'archetipo della apertura del mercato conferivano a questo potenzialità fondative di una "società aperta" anche al di là del terreno economico e si rivelavano capaci di favorire il superamento di chiusure radicate nella storia e nella cultura degli stati membri ${ }^{44}$. In una seconda stagione, avviatasi con l'art. 6 del TUE nel testo formulato a Maastricht e proseguita con la Carta di Nizza e le disposizioni di principio del progetto di trattato costituzionale, in gran parte confluite nel Trattato di Lisbona, sembra consolidarsi lo sforzo di costruire una assiologia dei diritti europei. Questa tendenza, chiaramente riflessa dalla struttura della Carta di Nizza, costruita su una griglia ed una gerarchia di valori fondamentali, non è stata priva di ricadute nella giurisprudenza comunitaria. Ne costituisce un esempio emblematico la sentenza Omega della Corte di giustizia del 2004. Nel dichiarare la compatibilità con il diritto comunitario del divieto di commercializzare in Germania giochi laser di simulazione di atti di violenza prodotti in altro stato membro, la Corte ha incentrato la motivazione sul riconoscimento della intangibilità della dignità dell'uomo come principio derivante dalle tradizioni costituzionali comuni e non solo come fondamento costituzionale interno di misure restrittive della libertà comunitaria di prestazione di servizi adottate dalla Germania per motivi di ordine pubblico ${ }^{45}$. Il riconoscimento della dignità dell'uomo come principio appartenente alle tradizioni costituzionali comuni, e quindi indipendentemente dal forte risalto di esso nell'ordinamento dello stato nel quale era sorta la controversia, va peraltro inquadrato nel contesto della tensione su ricordata fra neutralizzazione e assiologia dei diritti nell'ordinamento dell'UE. Una tensione della quale la Corte

\footnotetext{
${ }^{44}$ Il riferimento nel testo è a Corte di giustizia CE, causa C-159/1990, Society for the Protection of Unborn Children Ireland Ltd v. Grogan and others.

${ }_{45}$ Cfr. Corte di giustizia CE, 14 ottobre 2004, causa C-36/02, Omega Spielhallen- und Automatenaufstellung $\mathrm{GmBH}$ c. Oberbuergermeisterin der Bundesstadt Bonn.
} 
di giustizia sembra in qualche misura farsi carico, assegnando alla dignità dell'uomo la preferenza nella ponderazione con le libertà economiche, e dunque discostandosi dagli indirizzi che negano che l'intangibilità della dignità dell'uomo, in ragione del carattere assoluto che ne riveste la protezione, possa entrare in bilanciamento con diritti fondamentali o altri interessi costituzionalmente protetti $i^{46}$. Se questo approccio ridimensiona in qualche misura il forte significato del richiamo della giurisprudenza comunitaria ad un valore del tutto trascendente la sfera economica, non possono tacersi i rischi, potenzialmente costrittivi dell'identità "plurale" dei diritti in Europa, che possono derivare dall'accoglimento sul piano europeo di una concezione assoluta della dignità dell'uomo, sopratutto alla luce delle molteplici ricadute della intangibilità di essa su questioni di alto profilo etico che dividono profondamente la società europea.

Un secondo aspetto problematico riguarda il futuro dei diritti sociali in Europa, che resta ancora incerto fra un approdo minimale, che ne circoscrive la funzione a mere misure di salvaguardia degli standard di protezione assicurati negli stati membri, ed un approdo più ambizioso, che li assume come motore delle politiche sociali dell'Unione. Si gioca qui -mi sembra- una partita decisiva per il futuro dell'integrazione costituzionale in Europa. Non solo perché i destini di essa appaiono stretti fra la salvaguardia di un nucleo di garanzia di funzioni di riequilibrio socioeconomico, inseparabili dalla "statualità" e dalla fisionomia dello stato costituzionale democratico a partire dal XX secolo, e il quadro dei principi della "costituzione economica" dell'Unione, che lasciano ancora margini molto estesi alla copertura di politiche liberistiche di non intervento. Ma soprattutto perché la questione dei diritti sociali in Europa pone di fronte ad un alternativa che è centrale per la configurazione del completamento del processo di integrazione europea come un processo di integrazione costituzionale: quella fra il completo rinvio delle politiche sociali agli orizzonti ed ai compiti propri della statualità, e quella che comporta la presa d'atto della lezione che proviene dalle esperienze costituzionali dello stato liberale, la cui ispirazione liberistica riuscì a sorreggere le fasi di ascesa della borghesia capitalistica, ma non più a gestire le situazioni di crisi e le congiunture economiche.

Giunti alla conclusione, il discorso sull'ordinamento europeo dei diritti ritorna pertanto al nodo del significato begriffsgeschichtlich dell'integrazione. II percorso, al cui provvisorio odierno approdo sembra difficile rifiutare l'invito rivolto anni or sono to take rights seriously in Europa, occupa, nella ricostruzione della storia concettuale dell'integrazione, un posto decisivo, collocandosi al centro della transizione da una integrazione centrata sulla statualità, che ha una storia lunga, e non ancora esaurita, risalente al "sistema Westfalia", ad una mirata sulle dinamiche delle società pluralistiche.

\footnotetext{
${ }^{46}$ Sulla questione, molto dibattuta nella letteratura e nella giurisprudenza tedesca negli ultimi anni v., come posizioni rappresentative di due concezioni molto lontane della Menschenwürde, M. Herdegen, Art. 1 Abs. 1, in T. Maunz- G. Duerig, Grundgesetz Kommentar, München 2003; E. W. Böckenförde, Recht, Staat, Freiheit. Erweiterte Aufgabe, Frankfurt a. M. 2006, 379 ss.
} 
Le incertezze e le zone d'ombra che ho segnalato, le quali sono parte della gradualità del processo di costituzionalizzazione sovrastatuale in Europa, non autorizzano lo studioso ad anticipare conclusioni enfatiche, e a dimenticare il monito di Sir Isaiah Berlin, secondo il quale <poche azioni sono così pericolose per la causa della verità storica più del tentativo di trovare nella ghianda una quercia completamente sviluppata $>^{47}$. E tuttavia resta ferma, nell'itinerario che mi sono sforzato di tratteggiare, una lezione importante, che ha spinto ad avvicinarsi allo studio dell'integrazione europea come ad un grande laboratorio del ripensamento delle categorie del diritto pubblico dello stato nazione. Un approccio al quale la prospettiva dei diritti offre una chiave di lettura fondamentale, quella del superamento di una comprensione del legame fra diritti ed integrazione e della funzione di integrazione dei diritti tutta interna alla storia e ai destini dello stato nazionale. L'Europa ha posto e pone in misura crescente, in quanto scenario di migrazioni e di scambi, sfide inedite, che investono ad un tempo le basi dei diritti fondamentali e i meccanismi dell'integrazione sociale.

${ }^{47}$ Cfr. I. Berlin, Controcorrente. Saggi di storia delle idee, a cura di H. Hardy, Milano 2000, 36. 